\title{
The Rosser-Iwaniec sieve in number fields, with an application
}

\author{
by
}

M. D. Coleman (Manchester)

Introduction. In this paper we extend Iwaniec's results on Rosser's sieve to a general number field. The papers [13] and [14] of Iwaniec are long, but we can only be brief in justification of our extension. There have been many papers describing extensions of sieve methods to algebraic domains; Halberstam and Richert ([7], p. 340), give 24 references to such work. We will discuss briefly the relation of this paper to earlier ones at the end of Section 4.

As an application of the sieve we examine the distribution, in imaginary quadratic fields, of prime ideals that lie in sectors. As a corollary of our main result, we obtain

Theorem 1. Let $Q(x, y)$ be a positive definite, primitive, binary quadratic form with integer coefficients. Then there exist infinitely many primes $p$ with

$$
p=Q(m, n) \quad \text { and } \quad n<p^{0.1631}
$$

where $m, n \in \mathbb{Z}$.

The exponent 0.1631 is an improvement of the results (at least for $\left.Q(x, y)=x^{2}+y^{2}\right)$ of various authors. Kubilius, in a series of papers [20]-[22], proved that $12 / 29+\varepsilon$ is allowable, for all $\varepsilon>0$. This was improved to $1 / 3+\varepsilon$ by Bulota [2], $1 / 4+\varepsilon$ by Koval'chik [19] and $1 / 5+\varepsilon$ by S. Ricci in his Ph.D thesis, [26]. Conjecturally, assuming that the Riemann hypothesis holds for all Hecke $L$-functions with Grössencharaktere, over imaginary quadratic fields, Kubilius [22] and Ankeny [1] have shown that $Q(m, n)$ represents infinitely many primes $p$ with $n \ll \log p$. But further, the expectation that an irreducible polynomial in $\mathbb{Z}[x]$ should represent a prime infinitely often would lead us to conjecture that $Q(m, n)$ represents a prime infinitely often with $n=1$. This problem has also been studied by M. Maknys.

The Rosser sieve with error term as given by Iwaniec has had an important impact on the question of bounding the difference between consecutive 
rational primes, i.e. finding $\theta$ such that $p^{\prime}-p<p^{\theta}$ for all consecutive primes $p<p^{\prime}$. This question has been generalized in [3] and [5] as a question about the points at which norm-forms associated with number fields are prime. The details of applications of the sieve to questions of norm-forms will appear later. But this future application will serve as justification for presenting here the Rosser-Iwaniec sieve in general number fields.

1. The Rosser-Iwaniec sieve. Let $K$ be an algebraic number field of degree $n$, let $\mathfrak{a}, \mathfrak{b}, \mathfrak{c}, \ldots$ denote integral ideals of $K$ and $\mathfrak{p}, \mathfrak{q}$, prime ideals of $K$. The combinatorial aspect of a sieve requires some system such as a Dedekind Domain where we have unique factorization along with an order on the elements of the Domain. In the case of a number field, where the integral ideals have unique factorization, the order, $<_{K}$ say, will essentially be given by the norm $N_{K / \mathbb{Q}}$. That is, $N_{K / \mathbb{Q}}(\mathfrak{a})<N_{K / \mathbb{Q}}(\mathfrak{b})$ implies $\mathfrak{a}<_{K} \mathfrak{b}$. For ideals with equal norm the ordering can be arbitrary. The rule chosen here is that if $N \mathfrak{a}=N \mathfrak{b}$ and $a$ and $b$ are the ideal numbers of $\mathfrak{a}$ and $\mathfrak{b}$ respectively (see [10]), then $\mathfrak{a}<_{K} \mathfrak{b}$ iff $\arg a<\arg b$.

We now diverge from the normal set-up of sieves by defining $\mathcal{A}$ to be all the integral ideals of $K$ along with a weight function $\theta$ on the ideals. We define the cardinality of $\mathcal{A}$ to be

$$
|\mathcal{A}|=\sum_{\mathfrak{a} \in \mathcal{A}} \theta(\mathfrak{a}),
$$

which we assume to be finite. Further, for $\mathfrak{d}$ an ideal we define

$$
\left|\mathcal{A}_{\mathfrak{d}}\right|=\sum_{\substack{\mathfrak{a} \in \mathcal{A} \\ \mathfrak{d} \mid \mathfrak{a}}} \theta(\mathfrak{a})
$$

Let $\mathcal{P}$ be a subset of the prime ideals. Given any integral ideal $\mathfrak{h}$ and $z \geq 2$ our problem is to estimate the sifting functions

$$
S_{K}(\mathcal{A}, \mathcal{P}, \mathfrak{h})=\sum_{\substack{\mathfrak{a} \in \mathcal{A} \\\left(\mathfrak{a}, P_{K}(\mathfrak{h})\right)=1}} \theta(\mathfrak{a}) \text { and } S(\mathcal{A}, \mathcal{P}, z)=\sum_{\substack{\mathfrak{a} \in \mathcal{A} \\(\mathfrak{a}, P(z))=1}} \theta(\mathfrak{a})
$$

where

$$
P_{K}(\mathfrak{h})=\prod_{\substack{\mathfrak{p}<K \mathfrak{h} \\ \mathfrak{p} \in \mathcal{P}}} \mathfrak{p} \quad \text { and } \quad P(z)=\prod_{\substack{N \mathfrak{p}<z \\ \mathfrak{p} \in \mathcal{P}}} \mathfrak{p}
$$

2. Buchstab identities. Though it will be sufficient for applications to have bounds only for $S(\mathcal{A}, z)$ (where the dependence on $\mathcal{P}$ has been dropped) the necessity of looking at both forms of sifting function defined at (1.1) arises from our analogue of Buchstab's identity. This states that 


$$
\begin{aligned}
S_{K}(\mathcal{A}, \mathfrak{h}) & =|\mathcal{A}|-\sum_{\substack{\mathfrak{p}<K \mathfrak{h} \\
\mathfrak{p} \in \mathcal{P}}} S_{K}\left(\mathcal{A}_{\mathfrak{p}}, \mathfrak{p}\right), \\
S(\mathcal{A}, z) & =|\mathcal{A}|-\sum_{\substack{N \mathfrak{p}<z \\
\mathfrak{p} \in \mathcal{P}}} S_{K}\left(\mathcal{A}_{\mathfrak{p}}, \mathfrak{p}\right) .
\end{aligned}
$$

The proof of these identities follows from Legendre's formula in $K$,

$$
S_{K}(\mathcal{A}, \mathfrak{h})=\sum_{\mathfrak{d} \mid P_{K}(\mathfrak{h})} \mu(\mathfrak{d})\left|\mathcal{A}_{\mathfrak{d}}\right|
$$

as does

LEMMA 1.

$$
S_{K}(\mathcal{A}, \mathfrak{h})=\sum_{\mathfrak{d} \mid P_{K}(\mathfrak{h})} \mu(\mathfrak{d}) \varrho_{\mathfrak{d}}^{ \pm}\left|\mathcal{A}_{\mathfrak{d}}\right|+\sum_{\mathfrak{d} \mid P_{K}(\mathfrak{h})} \mu(\mathfrak{d}) \sigma_{\mathfrak{d}}^{ \pm} S_{K}\left(\mathcal{A}_{\mathfrak{d}}, \pi(\mathfrak{d})\right),
$$

where $\pi(\mathfrak{d})$ is the least (with respect to $<_{K}$ ) prime factor of $\mathfrak{d}$. The $\varrho_{\mathfrak{d}}^{ \pm}$and $\sigma_{\mathfrak{d}}^{ \pm}$can satisfy quite general conditions but here they are defined by

$$
\varrho_{(1)}^{ \pm}=1 \quad \text { and } \quad \sigma_{(1)}^{ \pm}=0,
$$

and for $\mathfrak{d}>_{K}(1)$, with the decomposition $\mathfrak{d}=\mathfrak{p}_{1} \ldots \mathfrak{p}_{r}, \mathfrak{p}_{1}>_{K} \ldots>_{K} \mathfrak{p}_{r}$,

$$
\varrho_{\mathfrak{d}}^{ \pm}=\prod_{1 \leq l \leq r} \lambda_{\mathfrak{p}_{1} \ldots \mathfrak{p}_{l}}^{ \pm} \quad \text { and } \quad \sigma_{\mathfrak{d}}^{ \pm}=\left(1-\lambda_{\mathfrak{p}_{1} \ldots \mathfrak{p}_{r}}^{ \pm}\right) \prod_{1 \leq l<r} \lambda_{\mathfrak{p}_{1} \ldots \mathfrak{p}_{l}}^{ \pm},
$$

where

$$
\lambda_{\mathfrak{d}}^{ \pm}=\lambda_{\mathfrak{d}}^{ \pm}(D)= \begin{cases}0 & \text { if } \mu(\mathfrak{d})= \pm 1 \text { and } N(\pi(\mathfrak{d}))>(D / N \mathfrak{d})^{1 / 2} \\ 1 & \text { otherwise. }\end{cases}
$$

Here $D$ is a real parameter and we sometimes show the dependence of $\varrho_{\mathfrak{d}}^{ \pm}$on $D$ by writing $\varrho_{\mathfrak{d}}^{ \pm}(D)$. There is the obvious analogue of Lemma 1 for $S(\mathcal{A}, z)$.

As in the rational case $\mu(\mathfrak{d}) \sigma_{\mathfrak{d}}^{+} \leq 0$ and $\mu(\mathfrak{d}) \sigma_{\mathfrak{d}}^{-} \geq 0$ for all $\mathfrak{d} \mid P_{K}(\mathfrak{h})$. So we have upper and lower bounds for $S_{K}(\mathcal{A}, \mathfrak{h})$ in terms of $\sum_{\mathfrak{d} \mid P_{K}(\mathfrak{h})} \mu(\mathfrak{d})$ $\times \varrho_{\mathfrak{d}}^{ \pm}(D)\left|\mathcal{A}_{\mathfrak{d}}\right|$. To obtain analogues of the results in [13] we could examine this latter sum, but to incorporate the innovations of [14] we "massage" the problem slightly.

Let $1 / 3>\varepsilon>0$ be given and $\mu=\varepsilon^{9}, u=D^{\varepsilon^{2}}$. Assume $u \leq z<D^{1 / 2}$, and given $\mathcal{A}$ and $\mathcal{P}$ define $\mathcal{A}^{*}=\{\mathfrak{a} \in \mathcal{A}:(\mathfrak{a}, P(u))=1\}(=S(\mathcal{A}, u))$, and $\mathcal{P}^{*}=\{\mathfrak{p} \in \mathcal{P}: \mathfrak{p} \nmid P(u)\}$. Then $S(\mathcal{A}, \mathcal{P}, z)=S\left(\mathcal{A}^{*}, \mathcal{P}^{*}, z\right)$, so by Lemma 1 ,

$$
\begin{aligned}
\sum_{\mathfrak{d} \mid P(z, u)} \mu(\mathfrak{d}) \varrho_{\mathfrak{d}}^{-}(D) S\left(\mathcal{A}_{\mathfrak{d}}, u\right)<S(\mathcal{A}, z) & \\
& <\sum_{\mathfrak{d} \mid P(z, u)} \mu(\mathfrak{d}) \varrho_{\mathfrak{d}}^{+}(D) S\left(\mathcal{A}_{\mathfrak{d}}, u\right),
\end{aligned}
$$


where $P(z, u)=P(z) / P(u)$. Denote the bounds in $(2.2)$ by $W^{ \pm}(\mathcal{A}, u, z)$ in the obvious manner. It is upper bounds for $W^{+}$and lower bounds for $W^{-}$ that are given in [14], where (2.2) is further improved by noting that for any $R \geq 1$ and $\varepsilon>0$ sufficiently small the $W^{ \pm}\left(\mathcal{A}, D^{\varepsilon^{2}}, z\right)$ are bounds for

$$
S(\mathcal{A}, z) \pm \sum_{\substack{\mathfrak{d} \mid P(z) \\ \Omega(\mathfrak{d}) \leq R}} \mu(\mathfrak{d}) \sigma_{\mathfrak{d}}^{ \pm}(D) S_{K}\left(\mathcal{A}_{\mathfrak{d}}, \pi(\mathfrak{d})\right),
$$

where $\Omega(\mathfrak{d})$ is the number of prime ideal factors of $\mathfrak{d}$.

Next, the interval $\left[u, D^{1 / 2}\right]$ is divided, as in [14], p. 315, into subintervals by points from $\mathcal{G}=\left\{D^{\varepsilon^{2}(1+\eta)^{n}}: n \geq 0\right\}$. If $\mathfrak{d}=\mathfrak{p}_{1} \ldots \mathfrak{p}_{r}, N \mathfrak{p}_{1}<D^{1 / 2}$, $\mathfrak{p}_{1}>_{K} \ldots>_{K} \mathfrak{p}_{r}$, and $N \mathfrak{p}_{r} \geq u$, we say that $\mathfrak{d}$ belongs to the sequence $\left(D_{1}, \ldots, D_{r}\right), D_{i} \in \mathcal{G}, i=1, \ldots, r$, iff

$$
D_{1} \leq N \mathfrak{p}_{1}<D_{1}^{1+\eta}, \quad \ldots, \quad D_{r} \leq N \mathfrak{p}_{r}<D_{r}^{1+\eta} .
$$

Looking at $W^{+}(\mathcal{A}, u, z)$, we bound from above by increasing the set of $\mathfrak{d}$ for which $\mu(\mathfrak{d}) \varrho_{\mathfrak{d}}^{+}$is positive while decreasing the set for which $\mu(\mathfrak{d}) \varrho_{\mathfrak{d}}^{+}$is negative. This first set of $\mathfrak{d}$ is contained in

$$
\begin{aligned}
\mathcal{D}_{1}^{+}=\mathcal{D}_{1}^{+}(D)=\{\mathfrak{d} \mid & P(z, u): \text { there exists } r \text { such that } \mathfrak{d} \text { belongs to } \\
& \left(D_{1}, \ldots, D_{2 r}\right) \text { where } D_{1} \geq D_{2} \geq \ldots \geq D_{2 r} \text { and } \\
& \left.D_{1} \ldots D_{2 l} D_{2 l+1}^{3}<D \text { for all } 0 \leq l<r\right\},
\end{aligned}
$$

while the second set contains

$$
\begin{aligned}
& \mathcal{D}_{2}^{+}(D)=\{\mathfrak{d} \mid P(z, u): \text { there exists } r \text { such that } \mathfrak{d} \text { belongs to } \\
&\left(D_{1}, \ldots, D_{2 r+1}\right) \text { where } D_{1}>D_{2}>\ldots>D_{2 r+1} \text { and } \\
&\left.D_{1} \ldots D_{2 l+1}^{3}<D^{1 /(1+\eta)} \text { for all } 0 \leq l \leq r\right\} .
\end{aligned}
$$

So

$$
W^{+}(\mathcal{A}, u, z) \leq S(\mathcal{A}, u)-\sum_{\mathfrak{d} \in \mathcal{D}_{2}^{+}} S\left(\mathcal{A}_{\mathfrak{d}}, u\right)+\sum_{\mathfrak{d} \in \mathcal{D}_{1}^{+}} S\left(\mathcal{A}_{\mathfrak{d}}, u\right)
$$

We can argue analogously for a lower bound of $W^{-}$where we decrease the set of $\mathfrak{d}$ for which $\mu(\mathfrak{d}) \varrho_{\mathfrak{d}}^{-}$is positive (to get $\mathcal{D}_{1}^{-}$), and increase the set of $\mathfrak{d}$ when it is negative (to get $\mathcal{D}_{2}^{-}$), obtaining then an obvious analogue of (2.3). We denote by $\mathcal{D}_{\text {diff }}^{+}$the set of $\mathfrak{d}$ that have either been introduced or removed from $W^{+}$above.

Applying to $(2.2)$ the lower and upper bounds of $S_{K}(\mathcal{A}, \mathfrak{h})$ that follow from Lemma 1 gives us

$$
W^{+}(\mathcal{A}, u, z) \leq \sum_{\mathfrak{q} \mid P(u)} \mu(\mathfrak{q}) \varrho_{\mathfrak{q}}^{+}\left(D^{\varepsilon}\right)\left|\mathcal{A}_{\mathfrak{q}}\right|
$$




$$
\begin{aligned}
& -\sum_{\mathfrak{q} \mid P(u)} \sum_{\mathfrak{d} \in \mathcal{D}_{2}^{+}} \mu(\mathfrak{q}) \varrho_{\mathfrak{q}}^{-}\left(D^{\varepsilon}\right)\left|\mathcal{A}_{\mathfrak{q} \mathfrak{d}}\right| \\
& +\sum_{\mathfrak{q} \mid P(u)} \sum_{\mathfrak{d} \in \mathcal{D}_{1}^{+}} \mu(\mathfrak{q}) \varrho_{\mathfrak{q}}^{+}\left(D^{\varepsilon}\right)\left|\mathcal{A}_{\mathfrak{q} \mathfrak{d}}\right| .
\end{aligned}
$$

Now we use the assumption fundamental to this sieve method, namely that $\left|\mathcal{A}_{\mathfrak{d}}\right|$ can be written in the form

$$
\left|\mathcal{A}_{\mathfrak{d}}\right|=\frac{\omega(\mathfrak{d})}{N \mathfrak{d}} X+r(\mathcal{A}, \mathfrak{d}),
$$

where $\frac{\omega(\mathfrak{d})}{N \mathfrak{d}} X$ is considered as a main term and $r(\mathcal{A}, \mathfrak{d})$ an error. Here, $\omega(\mathfrak{d})$ should be a multiplicative function and for each prime $\mathfrak{p} \in \mathcal{P}$ satisfy $0<\omega(\mathfrak{p})<N \mathfrak{p}$. It simplifies notation later if we define $\omega(\mathfrak{p})=0$ if $\mathfrak{p} \notin \mathcal{P}$.

Introducing this assumption into (2.4), we obtain

$$
\begin{aligned}
W^{+}(\mathcal{A}, u, z) & \leq\left\{\sum_{\mathfrak{q} \mid P(u)} \mu(\mathfrak{q}) \varrho_{\mathfrak{q}}^{+}\left(D^{\varepsilon}\right) \frac{\omega(\mathfrak{q})}{N \mathfrak{q}}-\sum_{\mathfrak{q} \mid P(u)} \sum_{\mathfrak{d} \in \mathcal{D}_{2}^{+}} \mu(\mathfrak{q}) \varrho_{\mathfrak{q}}^{-}\left(D^{\varepsilon}\right) \frac{\omega(\mathfrak{q} \mathfrak{d})}{N \mathfrak{q} \mathfrak{d}}\right. \\
& \left.+\sum_{\mathfrak{q} \mid P(u)} \sum_{\mathfrak{d} \in \mathcal{D}_{1}^{+}} \mu(\mathfrak{q}) \varrho_{\mathfrak{q}}^{+}\left(D^{\varepsilon}\right) \frac{\omega(\mathfrak{q} \mathfrak{d})}{N \mathfrak{q} \mathfrak{d}}\right\} X+\sum_{\substack{\mathfrak{q} \mid P\left(D^{\varepsilon^{2}}\right) \\
N \mathfrak{q}<D^{\varepsilon}}} \phi_{\mathfrak{q}}^{+}\left(D^{\varepsilon}\right) r(\mathcal{A}, \mathfrak{q}) \\
& +\sum_{\substack{\left(D_{1}, \ldots, D_{r}\right) \\
\in \mathcal{D}_{1}^{+} \cup \mathcal{D}_{2}^{+}}} \sum_{\substack{\mathfrak{q} \mid P\left(D^{\varepsilon^{2}}\right) \\
N \mathfrak{q}<D^{\varepsilon}}} \lambda_{\mathfrak{q}}^{+}\left(D_{1}, \ldots, D_{r}\right) \sum_{\substack{\mathfrak{d} \text { belongs to } \\
\left(D_{1}, \ldots, D_{r}\right)}} r(\mathcal{A}, \mathfrak{q} \mathfrak{d}),
\end{aligned}
$$

for some coefficients $\phi_{\mathfrak{q}}^{+}\left(D^{\varepsilon}\right)$ and $\lambda_{\mathfrak{q}}^{+}\left(D_{1}, \ldots, D_{r}\right)$, bounded by 1 in absolute value. The term on the right-hand side containing the factor $X$ will be considered as the main term.

3. Main term. We first transform the main term by replacing $\varrho_{\mathfrak{q}}^{-}\left(D^{\varepsilon}\right)$ with $\varrho_{\mathfrak{q}}^{+}\left(D^{\varepsilon}\right)$. Then on defining

$$
S^{ \pm}(D, \mathcal{P}, u)=\sum_{\mathfrak{q} \mid P(u)} \mu(\mathfrak{q}) \varrho_{\mathfrak{q}}^{ \pm}(D) \frac{\omega(\mathfrak{q})}{N \mathfrak{q}},
$$

the main term can be written as

$$
S^{+}\left(D^{\varepsilon}, \mathcal{P}, u\right)\left\{1-\sum_{\mathfrak{d} \in \mathcal{D}_{2}^{+}} \frac{\omega(\mathfrak{d})}{N \mathfrak{d}}+\sum_{\mathfrak{d} \in \mathcal{D}_{1}^{+}} \frac{\omega(\mathfrak{d})}{N \mathfrak{d}}\right\}+E_{1},
$$


where

$$
E_{1}=\left(S^{+}\left(D^{\varepsilon}, \mathcal{P}, u\right)-S^{-}\left(D^{\varepsilon}, \mathcal{P}, u\right)\right) \sum_{\mathfrak{d} \in \mathcal{D}_{2}^{+}} \frac{\omega(\mathfrak{d})}{N \mathfrak{d}} .
$$

We transform once more by replacing the sums over elements of $\mathcal{D}_{1}^{+}$and $\mathcal{D}_{2}^{+}$with the characteristic function $\mu(\mathfrak{d}) \varrho_{\mathfrak{d}}^{+}(D)$, when we get

$$
S^{+}\left(D^{\varepsilon}, \mathcal{P}, u\right)\left\{S^{+}\left(D, \mathcal{P}^{*}, z\right)+E_{2}\right\}+E_{1},
$$

with

$$
E_{2}=\sum_{\mathfrak{d} \in \mathcal{D}_{\text {diff }}^{+}} \frac{\omega(\mathfrak{d})}{N \mathfrak{d}} .
$$

It is from [13] that we obtain bounds not only for $S^{ \pm}(D, \mathcal{P}, z)$ but also $S_{K}^{ \pm}(D, \mathcal{P}, \mathfrak{h})$. Define

$$
V_{K}(\mathfrak{h})=\prod_{\mathfrak{p} \mid P_{K}(\mathfrak{h})}\left(1-\frac{\omega(\mathfrak{p})}{N \mathfrak{p}}\right)
$$

when

$$
\begin{aligned}
V_{K}(\mathfrak{h}) & =\sum_{\mathfrak{d} \mid P_{K}(\mathfrak{h})} \mu(\mathfrak{d}) \frac{\omega(\mathfrak{d})}{N \mathfrak{d}} \\
& =\sum_{\mathfrak{d} \mid P_{K}(\mathfrak{h})} \mu(\mathfrak{d}) \varrho_{\mathfrak{d}}^{ \pm}(D) \frac{\omega(\mathfrak{d})}{N \mathfrak{d}}+\sum_{\mathfrak{d} \mid P_{K}(\mathfrak{h})} \mu(\mathfrak{d}) \sigma_{\mathfrak{d}}^{ \pm}(D) \frac{\omega(\mathfrak{d})}{N \mathfrak{d}} V_{K}(\pi(\mathfrak{d})) .
\end{aligned}
$$

This last line follows in exactly the same way as Lemma 1 . The first term on the right-hand side is $S_{K}^{ \pm}(D, \mathcal{P}, \mathfrak{h})$, so we have

$$
S_{K}^{ \pm}(D, \mathcal{P}, \mathfrak{h})=V_{K}(\mathfrak{h}) \pm \sum_{r=(1 \mp 1) / 2}^{\infty} S_{r, \mathfrak{h}}^{ \pm}(D),
$$

where

$$
S_{r, \mathfrak{h}}^{+}(D)=\sum_{\substack{\mathfrak{p}_{2 r+1}<K \ldots<{ }_{K} \mathfrak{p}_{1}<K \mathfrak{h} \\ N\left(\mathfrak{p}_{2 l+1}^{3} \ldots \mathfrak{p}_{1}\right)<D, 0 \leq l<r \\ N\left(\mathfrak{p}_{2 r+1}^{3} \ldots \mathfrak{p}_{1}\right) \geq D}} \frac{\omega\left(\mathfrak{p}_{1} \ldots \mathfrak{p}_{2 r+1}\right)}{N\left(\mathfrak{p}_{1} \ldots \mathfrak{p}_{2 r+1}\right)} V_{K}\left(\mathfrak{p}_{2 r+1}\right),
$$

and similarly for $S_{r, \mathfrak{h}}^{-}(D)$, where the sum is over $2 r$-tuples, $\mathfrak{p}_{2 r}<_{K} \ldots<_{K}$ $\mathfrak{p}_{1}<_{K} \mathfrak{h}$, satisfying $N\left(\mathfrak{p}_{2 l}^{3} \mathfrak{p}_{2 l-1} \ldots \mathfrak{p}_{1}\right)<D, 0 \leq l<r$ and $N\left(\mathfrak{p}_{2 r}^{3} \mathfrak{p}_{2 r-1} \ldots \mathfrak{p}_{1}\right)$ $\geq D$. These are analogues of equations (4.1) and (4.2) in [13]. The interest in the present case will arise from the question of how conditions of the form 
$\mathfrak{p}<_{K} \mathfrak{h}$ relate with those of the form $N \mathfrak{p}<z$. Defining

$$
T_{R, \mathfrak{h}}^{ \pm}(D)=\sum_{r=(1 \mp 1) / 2}^{R} S_{r, \mathfrak{h}}^{ \pm}(D) \quad \text { for } \quad \frac{\log D}{\log N \mathfrak{h}}>\frac{3 \mp 1}{2},
$$

we look for upper bounds as $R \rightarrow \infty$. It should be noted that analogues of the above, namely $S_{r, z}^{ \pm}(D)$ and $T_{R, z}^{ \pm}(D)$ are formed by replacing $\mathfrak{p}_{1}<_{K} \mathfrak{h}$ with $N \mathfrak{p}_{1}<z$.

We now introduce the assumption that there exists a constant $\kappa \geq 2$ such that for all $\mathfrak{j}>_{K} \mathfrak{h}, N \mathfrak{h} \geq 2$ we have

$$
\prod_{\mathfrak{h} \leq K \mathfrak{p}<_{K} \mathfrak{j}}\left(1-\frac{\omega(\mathfrak{p})}{N \mathfrak{p}}\right)^{-1}<\left(\frac{\log N \mathfrak{j}}{\log N \mathfrak{h}}\right)\left(1+\frac{\kappa}{\log N \mathfrak{h}}\right) .
$$

In the terminology of sieves, we say we have a "linear sieve", because the ratio $\log N \mathfrak{j} / \log N \mathfrak{h}$ occurs to the first power. Looking first at $R=0$ we have

$$
T_{0, \mathfrak{h}}^{+}(D)=S_{0, \mathfrak{h}}^{+}(D)=\sum_{\substack{\mathfrak{p}<K \mathfrak{h} \\ N \mathfrak{p} \geq D^{1 / 3}}} \frac{\omega(\mathfrak{p})}{N \mathfrak{p}} V_{K}(\mathfrak{p})=V_{K}\left(D^{1 / 3}\right)-V_{K}(\mathfrak{h}) .
$$

This is zero if there is no prime $\mathfrak{p}$ such that $N \mathfrak{p} \geq D^{1 / 3}$ and $\mathfrak{p}<_{K} \mathfrak{h}$. Otherwise it equals

$$
\begin{gathered}
V_{K}(\mathfrak{h})\left\{\frac{V_{K}\left(D^{1 / 3}\right)}{V_{K}(\mathfrak{h})}-1\right\} \\
\quad<V_{K}(\mathfrak{h})\left\{\left(\frac{\log N \mathfrak{h}}{\log D^{1 / 3}}\right)\left(1+\frac{3 \kappa}{\log D}\right)-1\right\} \\
=V_{K}(\mathfrak{h}) s^{-1}\left\{3-s+\frac{3 \kappa}{\log D}\right\},
\end{gathered}
$$

on writing $s=\log D / \log N \mathfrak{h}$. The condition that there exists a prime $\mathfrak{p}$ satisfying $N \mathfrak{p} \geq D^{1 / 3}, \mathfrak{p}<_{K} \mathfrak{h}$ implies $N \mathfrak{h} \geq D^{1 / 3}$, i.e. $s \leq 3$, in which range the $3-s$ occurring in (3.4) equals $T_{0}^{+}(s)$ as defined in [13], p. 193. We can note here a difference between the present situation and Iwaniec's. In [13] when $s=3, T_{0, z}^{+}(y)$ is necessarily zero, yet for $T_{0, \mathfrak{h}}^{+}(D)$, even if $s=3$, there may exist a prime $\mathfrak{p}$ such that $N \mathfrak{p} \geq D^{1 / 3}$ and $\mathfrak{p}<_{K} \mathfrak{h}$ when $T_{0, \mathfrak{h}}^{+}(D)$ would not be zero. So the $3 \kappa / \log D$ in (3.4) is necessary.

We need to show that a bound such as (3.4) holds for all $R$. To see what the error should be in general we look at the limited ranges of $D$ and $s$ in which $T_{R, \mathfrak{h}}^{ \pm}(D)$ can be estimated directly from (3.2). We note that $S_{r, \mathfrak{h}}^{+}(D)$ (respectively $S_{r, \mathfrak{h}}^{-}(D)$ ) is zero if there exist no set of primes satisfying $N\left(\mathfrak{p}_{2 r+1}^{3} \ldots \mathfrak{p}_{1}\right)>D$ and $\mathfrak{p}_{2 r+1}<_{K} \ldots<_{K} \mathfrak{p}_{1}<_{K} \mathfrak{h}$ (no set satisfying 
$N\left(\mathfrak{p}_{2 r}^{3} \ldots \mathfrak{p}_{1}\right)>D$ and $\left.\mathfrak{p}_{2 r}<_{K} \ldots<_{K} \mathfrak{p}_{1}<_{K} \mathfrak{h}\right)$, which is certainly satisfied if $D>N \mathfrak{h}^{2 r+3}$, i.e. $s>2 r+3(s>2 r+2)$. For the non-zero $S_{r, \mathfrak{h}}^{ \pm}(D)$, use $V_{K}(\mathfrak{p}) \leq 1$, drop the conditions on the norms and bound as

$$
S_{r, \mathfrak{h}}^{+}(D) \leq \frac{1}{(2 r+1) !}\left(\sum_{\mathfrak{p}<K} \frac{\omega(\mathfrak{p})}{N \mathfrak{h}}\right)^{2 r+1} ;
$$

the bound for $S_{r, \mathfrak{h}}^{-}(D)$ having $2 r$ in place of $2 r+1$. Next observe that

$$
\sum_{\mathfrak{p}<K \mathfrak{h}} \frac{\omega(\mathfrak{p})}{N \mathfrak{p}}>\log V_{K}(\mathfrak{h})>(2 \kappa \log N \mathfrak{h})^{-1},
$$

the latter inequality coming from (3.3). But now we have the same bounds for $T_{R, \mathfrak{h}}^{ \pm}(D)$ as Iwaniec has for his $T_{R, z}^{ \pm}(s)$ with $N \mathfrak{h}$ replacing $z$. Thus we can follow p. 198 of [13] to deduce

$$
T_{R, \mathfrak{h}}^{ \pm}(D)<V_{K}(\mathfrak{h}) s^{-1} G_{N \mathfrak{h}}^{ \pm}(s)(\log D)^{-1 / 3},
$$

if $\kappa^{24} \gg \log D$ or $s^{50} \geq \log D(\log \log D)^{3}$, where $G_{N \mathfrak{h}}^{ \pm}(s)$ is defined on p. 197 of [13]. The right-hand side of (3.5) will replace the $3 \kappa / \log D$ in (3.4) for general $R$. To find the replacement for the $3-s$, or $T_{0}^{+}(s)$ in (3.4), look just at the range of $D$ and $s$ such that $\kappa^{-24} \log D$ is sufficiently large and $s<s_{0}=(\log D)^{1 / 50}(\log \log D)^{3 / 50}$ and use the equations

$$
T_{R, \mathfrak{h}}^{ \pm}(D)=T_{R, D^{1 / s_{0}}}^{ \pm}(D)+\sum_{\substack{\mathfrak{p}<K \mathfrak{h} \\ D^{1 / s_{0}} \leq N \mathfrak{p}}} \frac{\omega(\mathfrak{p})}{N \mathfrak{p}} T_{R-1+(1 \pm 1) / 2, \mathfrak{p}}^{\mp}\left(\frac{D}{N \mathfrak{p}}\right)
$$

This is valid for $T^{-}$when $s \geq 2$ and for $T^{+}$if $S_{0, \mathfrak{h}}^{+}(D)=0$, i.e. $\mathfrak{h}$ and $D$ are such that there does not exist a prime satisfying $\mathfrak{p}<_{K} \mathfrak{h}$ and $N \mathfrak{p} \geq D^{1 / 3}$. If such a prime does exist, write

$$
T_{R, \mathfrak{h}}^{+}(D)=T_{0, \mathfrak{h}}^{+}(D)+T_{R, D^{1 / 3}}^{+}(D) .
$$

We have a bound for $T_{0, \mathfrak{h}}^{+}(D)$ in (3.7), while we will be able to bound the second term if we have results for $T_{R, z}^{+}(D)$. Such results will not be given explicitly, but the bounds for $T_{R, z}^{ \pm}(D)$ will be totally analogous to those of $T_{R, \mathfrak{h}}^{ \pm}(D)$ on replacing $N \mathfrak{h}$ by $z$ in the results. So we can use (3.6) to give bounds on $T_{R, \mathfrak{h}}^{+}(D)$ for $s \geq 3$, and the $s=3$ case can be used on the second term of (3.7).

The sum in (3.6) allows the use of induction, the hypothesis being that

$$
T_{R, \mathfrak{h}}^{ \pm}(D) \leq V_{K}(\mathfrak{h}) s^{-1}\left\{T_{R}^{ \pm}(s)+G_{N \mathfrak{h}}(s)(\log D)^{-1 / 3}\right\},
$$

for some $T_{R}^{ \pm}(s)$ and $G_{N \mathfrak{h}}(s)$. All we have so far is that $T_{0}^{+}(s)=3-s$ for $s \leq 3$ and 0 for $s>3$. But the use of $T_{0}^{+}(s)$ in (3.6) will lead to the same 
$T_{R}^{ \pm}(s)$ as Iwaniec has in [13]. This is because from (3.5) we can deduce an exact analogue of Lemma 21 of [13], namely:

Let $B(x)$ be a positive, continuous and increasing function in the interval $w \leq x \leq N \mathfrak{h}$. Then

$$
\sum_{\substack{w \leq N \mathfrak{p} \\ \mathfrak{p}<K}} \frac{\omega(\mathfrak{p})}{N \mathfrak{p}} \frac{V_{K}(\mathfrak{p})}{V_{K}(\mathfrak{h})} \frac{\log N \mathfrak{p}}{\log N \mathfrak{h}} B(N \mathfrak{p}) \leq \int_{w}^{N \mathfrak{h}} B(t) d(\log \log t)+3 \kappa \frac{B(N \mathfrak{h})}{\log w}
$$

subject to (3.3). Substituting the first term from (3.8) into the sum in (3.6) and using (3.9) gives a term

$$
\int_{s}^{s_{0}}\left(1-\frac{1}{t}\right)^{-1} T_{R-1+(1 \pm 1) / 2}^{\mp}(t-1) \frac{d t}{t}
$$

which, when the integral is completed up to infinity, would need to equal $T_{R}^{ \pm}(s)$ for the induction to work. This equality is exactly the recursive definition of $T_{R}^{ \pm}(s)$ as given in equation (7.3) of [13]. The properties of $T_{R}^{ \pm}(s)$ and $T^{ \pm}(s)=\lim _{R \rightarrow \infty} T_{R}^{ \pm}(s)$ are derived in [13].

Because the $T_{R}^{ \pm}(s)$ in (3.8) are identical to those in Iwaniec's work so are the conditions on $G_{N \mathfrak{h}}^{ \pm}(s)$, necessary for it to satisfy the induction step as well as (3.5). Thus we can take as $G_{N \mathfrak{h}}^{ \pm}(s)$ in (3.8) the function defined on p. 197 of [13].

Substituting (3.8) into (3.2), letting $R \rightarrow \infty$ and quoting the properties of $G_{N \mathfrak{h}}^{ \pm}(s)$ from [13] we have

LEMma 2. If condition (3.3) holds, then

$$
S_{K}^{+}(D, \mathcal{P}, \mathfrak{h})<V_{K}(\mathfrak{h})\left\{F(s)+O\left(e^{\sqrt{\kappa}-s}(\log D)^{-1 / 3}\right)\right\} \quad \text { if } N \mathfrak{h} \leq D,
$$

and

$$
S_{K}^{-}(D, \mathcal{P}, \mathfrak{h})>V_{K}(\mathfrak{h})\left\{f(s)+O\left(e^{\sqrt{\kappa}-s}(\log D)^{-1 / 3}\right)\right\} \quad \text { if } N \mathfrak{h} \leq D^{1 / 2},
$$

where $F(s)=1+T^{+}(s)=1+O\left(e^{-s}\right)$ and $f(s)=1-T^{-}(s)=1+O\left(e^{-s}\right)$ are the continuous solutions of the linear difference-differential equations given as (1.8) and (1.9) of [13].

Lemma 2 and (3.3) are sufficient to deal with all terms in (3.1) apart from $E_{2}$. The conditions for inclusion of an ideal into $\mathcal{D}_{\text {diff }}^{+}$depend on the norms of the prime divisors of the ideal and not on the position of the primes in the ordering defined by $<_{K}$. Thus we can follow Iwaniec [14], pp. 318-319, by assuming that there exists a constant $L>1$ such that

$$
\sum_{\substack{w<N \mathfrak{p}<z \\ \mathfrak{p} \in \mathcal{P}}} \sum_{a \geq 2} \frac{\omega\left(\mathfrak{p}^{a}\right)}{N \mathfrak{p}^{a}} \leq \frac{L}{\log 3 w},
$$


for all $z>w>2$, and deducing $E_{2} \ll \varepsilon^{3}+\varepsilon^{-6} \kappa^{3} e^{L}(\log D)^{-1}$. Using this bound on $E_{2}$ along with Lemma 2 we obtain from (2.6) (and the corresponding lower bound for $\left.W^{-}(\mathcal{A}, u, z)\right)$

Lemma 3 (Rosser's sieve). Let $\varepsilon>0, D \geq 2, z<D^{1 / 2}$. If (3.3) and (3.10) hold, then

$$
\begin{aligned}
& S(\mathcal{A}, z) \leq W^{+}\left(\mathcal{A}, D^{\varepsilon^{2}}, z\right) \leq V(z) X\{F(s)+E\}+R^{+}(\mathcal{A}, D), \\
& S(\mathcal{A}, z) \geq W^{-}\left(\mathcal{A}, D^{\varepsilon^{2}}, z\right) \geq V(z) X\{f(s)-E\}-R^{-}(\mathcal{A}, D),
\end{aligned}
$$

where $s=(\log D) / \log z, E \ll \varepsilon+\varepsilon^{-8} e^{\kappa+L}(\log D)^{-1 / 3}$ and $R^{+}(\mathcal{A}, D)$ denotes the last two sums in (2.6), $R^{-}$being defined similarly.

In fact, we can only use (2.6) for $D^{\varepsilon^{2}}<z$, but for smaller $z$ we can use the argument on p. 314 of [14], which depends only on the properties of $F$ and $f$. In applications $E$ is often taken as $c \varepsilon+O\left((\log D)^{-1 / 3}\right)$ for some $c>0$.

4. The error terms, $R^{ \pm}$. The errors in (2.6) are identical in form to those in Theorem 4 of [14]. In particular, the conditions for the sequence $\left(D_{1}, \ldots, D_{r}\right)$ to occur in $(2.6)$ are the same as in [14]. So we may use Lemma 1 of [14], simply a result on admissible $\left(D_{1}, \ldots, D_{r}\right)$, to rewrite the errors $R^{ \pm}$as bilinear sums. Lemma 1 of [14] is used to replace the sum over ideals $\mathfrak{d}$ belonging to $\left(D_{1}, \ldots, D_{r}\right)$ in $(2.6)$ by sums over ideals $\mathfrak{a}, \mathfrak{b}$ whose product belongs to $\left(D_{1}, \ldots, D_{r}\right)$. The importance of the lemma is that it allows great freedom in the ranges of $\mathfrak{a}$ and $\mathfrak{b}$, i.e. $N \mathfrak{a}<A, N \mathfrak{b}<B$ for any $A, B>1$, $A B=D^{1+\eta}$. Combining the sum over $\mathfrak{q} \mid P\left(D^{\varepsilon^{2}}\right), N \mathfrak{q}<D^{\varepsilon}$ in (2.6) with that over $\mathfrak{a}$, we have a bilinear sum for each admissible $\left(D_{1}, \ldots, D_{r}\right)$ of lengths $A D^{\varepsilon}$ and $B$. We can follow p. 312 of [14] in reinterpreting the parameters so that the sums are of length $A$ and $B$ with $A B=D$. That the main terms of Lemma 3 are identical to Iwaniec's means that the reinterpretation leaves them unchanged in form. There is a reduction in the range of validity of Lemma 3 to $z<D^{1 / 2(1+\varepsilon+\eta)}$ but this can be dealt with as in [14] because we have the Buchstab identities in (2.1).

Thus

$$
R^{ \pm}(\mathcal{A}, D)=\sum_{1 \leq l<\exp \left(8 \varepsilon^{-3}\right)} R_{l}^{ \pm}
$$

where

$$
R_{l}^{ \pm}=\sum_{\substack{N \mathfrak{a}<A, N \mathfrak{b}<B \\ \mathfrak{a} \mathfrak{b} \mid P(z)}} a^{ \pm}(\mathfrak{a}) b^{ \pm}(\mathfrak{b}) r(\mathcal{A}, \mathfrak{a} \mathfrak{b}),
$$

and the $a^{ \pm}(\mathfrak{a}), b^{ \pm}(\mathfrak{b})$ depend on $A, B, l$ and $\varepsilon$ and are bounded by 1 in absolute value. 
When sieving rational integers in a linear problem, the Rosser sieve gives the same results as the iterated Selberg sieve (see [7], Theorem 8.3). By way of two examples, we show this is true also in number fields.

The first time the iterated Selberg sieve appears, along with the associated functions $f$ and $F$, is in Jurkat and Richert, [17]. The sieve and an application from this paper are generalized by Schaal, [29]. Assume $K$ is a number field of degree $n=r_{1}+2 r_{2}$ (in the usual notation) with integers $\mathfrak{O}_{K}$. Let $y_{1}, \ldots, y_{n}$ be positive real numbers and set $y=y_{1} \ldots y_{n}$. Define

$$
\begin{array}{r}
\mathcal{R}=\mathcal{R}\left(y_{1}, \ldots, y_{n}\right)=\left\{\alpha \in \mathfrak{O}_{K}: 0<\alpha^{(i)}<y_{i}, i=1, \ldots, r_{1},\right. \\
\left.\left|\alpha^{(i)}\right|<y_{i}, i=r_{1}+1, \ldots, n\right\} .
\end{array}
$$

Then, for $\beta \in \mathfrak{O}_{K}$ and integral ideal $\mathfrak{f}$ with $(\beta, \mathfrak{f})=1$, Schaal generalizes the iterated Selberg sieve to give bounds for

$$
\left|\left\{\alpha \in \mathcal{R}\left(y_{1}, \ldots, y_{n}\right): \alpha \equiv \beta \bmod \mathfrak{f},\left(\alpha-\beta, V_{K}(z)\right)=1\right\}\right| .
$$

Using a weighted sum due to Kuhn that can be expressed as a linear sum of terms (4.2) with various $y_{i}$ and $\mathfrak{f}$, it can be deduced that given $\varepsilon>0$, $N \mathfrak{f}$ sufficiently large and any $y>N \mathfrak{f}^{25 / 11+\varepsilon}$, there exist $\zeta \in \mathfrak{O}_{K}$ with $\zeta \equiv$ $\beta \bmod \mathfrak{f}, \zeta \in \mathcal{R}\left(y_{1}, \ldots, y_{n}\right)$ and the number of prime factors of $\zeta$ at most two. No doubt 25/11 can be improved using Richert's weights (see [27] and also Theorem 9.6 of [7]). But here we remark that bounds can be given for (4.2) using Lemma 3. Define

$$
\theta(\mathfrak{a})=\left|\left\{\alpha \in \mathcal{R}\left(y_{1}, \ldots, y_{n}\right): \alpha \equiv \beta \bmod \mathfrak{f},(\alpha-\beta)=\mathfrak{a}\right\}\right| .
$$

The main term for $\sum_{\mathfrak{o} \mid \mathfrak{a}} \theta(\mathfrak{a})$ is given by Rieger [28], with $\omega(\mathfrak{p})=N \mathfrak{p} / \phi(\mathfrak{p})$ if $\mathfrak{p} \nmid \beta$, zero otherwise. Then (3.3) follows from Mertens's Theorem in $K$ (see (7.2)). Thus we can apply Lemma 3, obtaining the same bounds as Schaal.

As another example we might mention Hinz's proof ([12]) that in a totally real algebraic number field, every totally positive even algebraic integer $\zeta$ with sufficiently large norm can be represented as $\zeta=\omega+\Pi_{2}$ where $\omega$ is prime and $\Pi_{2}$ has at most two prime factors. (An algebraic integer is even if it is divisible by all prime ideals that divide 2.) In this case it is necessary to bound

$$
\left|\left\{\omega \in \mathcal{R}\left(y_{1}, \ldots, y_{n}\right): \omega \equiv \beta \bmod \mathfrak{q},\left(\omega-\beta, V_{K}(z)\right)=1\right\}\right| .
$$

As for the first problem, define

$$
\theta(\mathfrak{a})=\left|\left\{\omega \in \mathcal{R}\left(y_{1}, \ldots, y_{n}\right): \omega \equiv \beta \bmod \mathfrak{q},(\omega-\beta)=\mathfrak{a}\right\}\right| .
$$

Then the main term for $\sum_{\mathfrak{d} \mid \mathfrak{a}} \theta(\mathfrak{a})$ is given by the prime number theorem in Mitsui [23], with $\omega(\mathfrak{p})=N \mathfrak{p} / \phi(\mathfrak{p})$ if $\mathfrak{p} \nmid \beta$, zero otherwise. Thus Lemma 3 can be applied. The errors in Lemma 3 are dealt with in this problem by Hinz's extension of Bombieri's prime number theorem ([11]). 
5. Main result. Let $\mathfrak{f}$ be an integral ideal in the imaginary quadratic field $k$. Let $I_{\mathfrak{f}}$ be the group of fractional ideals whose prime decomposition contains no prime factors of $\mathfrak{f}$, and let

$$
P_{\mathfrak{f}}=\left\{(\alpha) \in I_{\mathfrak{f}}: \alpha \in k^{*}, \alpha \equiv 1 \bmod \mathfrak{f}\right\} .
$$

A Grössencharaktere on $I_{\mathfrak{f}}$ has the form $\lambda^{m}$, where $m \in \mathbb{Z}$ and, for $(\alpha) \in P_{\mathfrak{f}}, \lambda((\alpha))=(\alpha /|\alpha|)^{\omega(\mathfrak{f})}$, with $\omega(\mathfrak{f})$ the number of units $\varepsilon$ satisfying $\varepsilon \equiv 1 \bmod \mathfrak{f}$. (See [3], Section 2, for further details.) For $\mathfrak{a} \in I_{\mathfrak{f}}$ define $\psi(\mathfrak{a}) \in[0,1)$ by $\lambda(\mathfrak{a})=\exp (2 \pi i \psi(\mathfrak{a}))$. Let $I$ be an ideal class $\bmod \mathfrak{f}$, i.e. an element of $I_{\mathfrak{f}} / P_{\mathfrak{f}}$. Then our main result is

Theorem 2. For $x^{-1 / 3} \log ^{3} x>l \geq x^{-0.33691}$ we have

$$
\sum_{\substack{\mathfrak{p} \in I \\\left|\psi(\mathfrak{p})-\psi_{0}\right|<l \\ x<N \mathfrak{p}<2 x}} 1 \geq \frac{1}{500} \frac{2 l x}{h(\mathfrak{f}) \log x},
$$

for all $\psi_{0} \in[0,1), x>x_{0}$. Here $h(\mathfrak{f})$ is the order of $I_{\mathfrak{f}} / P_{\mathfrak{f}}$.

To deduce Theorem 1 write $Q(x, y)=a x^{2}+b x y+c y^{2}$ for some $a, b, c \in \mathbb{Z}$, and note that positive definite implies $b^{2}-4 a c=d f^{2}<0$ where $d$ is the discriminant of the imaginary quadratic field $k$. Associated with the form is a $\mathbb{Z}$-module $M=[a,(b-f \sqrt{d}) / 2] \subseteq \mathfrak{O}_{k}$. So if $\alpha(x, y)=a x+\left(\frac{b-f \sqrt{d}}{2}\right) y$, then $Q(x, y)=a^{-1} N(\alpha(x, y))$. We are assuming that $Q$ is primitive so it is, in fact, an example of a value-primitive, full-norm polynomial as defined in [24]. We now use ideas implicit in Section 5 of [5].

In generality let $K$ be a number field of degree $n, M \subseteq \mathfrak{O}_{K}$ a full module and $\gamma \in \mathfrak{O}_{K}$. Define $J$ to be the largest positive divisor of the rational integers $N(\gamma+\eta), \eta \in M$. Then from the proof of Proposition 5 of [5] we have that there exist integral ideals $\mathfrak{d}, \mathfrak{f}$ and $I \in I_{\mathfrak{f}} / P_{\mathfrak{f}}$ such that $N \mathfrak{d}=J$ and if $\mathfrak{a} \in I$ then $\mathfrak{a} \mathfrak{d}=(\gamma+\eta)$ for some $\eta \in M$. Further, if $(\mathfrak{d}, \mathfrak{f}) \neq(1)$ then, from the proof of Theorem 6 of [5] we have that there exist integral ideals $\mathfrak{e}, \mathfrak{f}^{\prime}$ satisfying $(\mathfrak{e}, \mathfrak{f})=(1)$ and $\mathfrak{f}^{\prime} \mid \mathfrak{f}$, along with $s, t \in \mathfrak{O}_{K}$ and $I^{\prime} \in I_{\mathfrak{f}^{\prime}} / P_{\mathfrak{f}^{\prime}}$ such that if $\mathfrak{a} \in I^{\prime}$ then $(t) \mathfrak{e} \mathfrak{a}=(s)(\gamma+\eta)$ for some $\eta \in M$. This has the advantage that if $\lambda$ is a Grössencharaktere $\bmod \mathfrak{f}^{\prime}$ on $I_{\mathfrak{f}^{\prime}}$, then $\lambda(\mathfrak{e})$ and $\lambda(\mathfrak{a})$ are well-defined. In this case $J=N((t) \mathfrak{e} /(s))$.

In the particular number field $k$ arising from $Q(x, y)$ we have $\gamma=0$, $J=a$ and we can find $\mathfrak{e}, \mathfrak{f}^{\prime}, I^{\prime}, s$ and $t$ as above. Apply Theorem 2 with $\psi$ defined as earlier $\bmod \mathfrak{f}^{\prime}$ and $\psi_{0}=\psi(s)-\psi(t)-\psi(\mathfrak{e})$. The number of prime ideals satisfying $x<N \mathfrak{p}<2 x$ and $N \mathfrak{p}=p^{2}$ is $\ll x^{1 / 2}$ so we can assume Theorem 2 counts only $\mathfrak{p}$ such that $N \mathfrak{p}=p$, prime. For these $\mathfrak{p} \in I^{\prime}$ we have $(t) \mathfrak{p} \mathfrak{e}=(s)(\eta)$ for some $\eta \in M$, so $\psi(t)+\psi(\mathfrak{p})+\psi(\mathfrak{e})=\psi(s)+\psi(\eta)$. The condition $\left|\psi(\mathfrak{p})-\psi_{0}\right|<l$ then becomes $|\psi(\eta)|<l$ which we rewrite as $\arg \eta<2 \pi \omega\left(\mathfrak{f}^{\prime}\right) x^{-\theta}$ where $x^{-\theta}=l \geq x^{-0.33691}$, i.e. $\theta \leq 0.33691$. But 
$\eta \in M$ if, and only if, $\eta=\alpha(m, n)$ for some $m, n \in \mathbb{Z}$ and so $p=N \mathfrak{p}=$ $N((s) /(t) \mathfrak{e}) N \eta=a^{-1} N(\alpha(m, n))=Q(m, n)$. Also, $\arg \eta<2 \pi \omega\left(\mathfrak{f}^{\prime}\right) x^{-\theta}$ implies

$$
n<C_{1} x^{-\theta} N(\alpha(m, n))^{1 / 2}<C_{1}\left(\frac{N \mathfrak{p}}{2}\right)^{-\theta}(a N \mathfrak{p})^{1 / 2}=C_{2} p^{1 / 2-\theta},
$$

for some $C_{1}>0$. Combining the intervals $(x, 2 x)$ we obtain

COROllary 4. There exists $C=C(Q)$ such that for all $\theta<0.3369$ and all $x>x_{0}$ the cardinality of

$$
\left\{p<x: p=Q(m, n), m, n \in \mathbb{Z} \text { and } n<C p^{1 / 2-\theta}\right\}
$$

is $\gg x^{1-\theta} / \log x$.

Theorem 1 follows immediately.

6. Introduction of sieve and asymptotic results. As in [3], Section 4 , or [5], Section 3, we start with

$$
\sum_{\substack{\mathfrak{p} \in I \\ x<N \mathfrak{p}<2 x \\\left|\psi(\mathfrak{p})-\psi_{0}\right|<l}} 1 \geq \sum_{\mathfrak{p} \in I} g(N \mathfrak{p}) f\left(\psi(\mathfrak{p})-\psi_{0}\right),
$$

where $f$ and $g$ are smooth functions with values between 0 and 1 , such that $f(t)$ is periodic mod 1 and equals 1 for $|t|<l-\Delta_{1}$ and 0 for $1 / 2 \geq|t|>l$ while $g(t)$ equals 1 for $x\left(1+\Delta_{2}\right)<t<2 x\left(1-\Delta_{2}\right)$ and 0 for $t<x$ or $t>2 x$. We in fact choose $\Delta_{1}=l / \log x$ and $\Delta_{2}=1 / \log x$, and the required properties of $f$ and $g$ will be quoted from [3] or [5] as necessary.

Define $\theta(\mathfrak{a})=g(N \mathfrak{a}) f\left(\psi(\mathfrak{a})-\psi_{0}\right)$ if $\mathfrak{a} \in I$, zero otherwise. Then the lower bound in (6.1) equals

$$
\sum_{\mathfrak{p}} \theta(\mathfrak{p})=S\left(\mathcal{A}, x^{1 / 2}\right)=S(\mathcal{A}, z)-\sum_{z<N \mathfrak{q}<x^{1 / 2}} S\left(\mathcal{A}_{\mathfrak{q}}, \mathfrak{q}\right)
$$

using Buchstab. Here $z$ is to be chosen. Set

$$
S(Q)=\sum_{Q \leq N \mathfrak{q}<2 Q} \sum_{\mathfrak{p}} \theta(\mathfrak{p q})
$$

with the inner sum over prime ideals. If $Q>x^{1 / 3}$ then this equals $\sum_{Q \leq N \mathfrak{q}<2 Q} S\left(\mathcal{A}_{\mathfrak{q}}, \mathfrak{q}\right)$. Now

$$
\begin{aligned}
S(Q) & =\sum_{Q \leq N \mathfrak{q}<2 Q} \sum_{\mathfrak{a}} \frac{\Lambda(\mathfrak{a})}{\log N \mathfrak{a}} \theta(\mathfrak{a q q})+E \\
& =\left(1+O\left(\frac{1}{\log x}\right)\right) \sum_{Q \leq N \mathfrak{q}<2 Q} \frac{1}{\log x / N \mathfrak{q}} \sum_{\mathfrak{a}} \Lambda(\mathfrak{a}) \theta(\mathfrak{a} \mathfrak{q})+E .
\end{aligned}
$$


Here $\Lambda$ is von Mangoldt's function, so $\Lambda(\mathfrak{a})=\log N \mathfrak{p}$ if $\mathfrak{a}$ is a power of the prime ideal $\mathfrak{p}, 0$ otherwise, and

$$
E=\sum_{Q \leq N \mathfrak{q}<2 Q} \sum_{j \geq 2} \sum_{\substack{x / N \mathfrak{q}<N \mathfrak{a}^{j}<2 x / N \mathfrak{q} \\\left|\psi\left(\mathfrak{a}^{j} \mathfrak{q}\right)-\psi_{0}\right|<l}} 1 .
$$

For $j \geq 3$ we can drop the condition on $\psi(\mathfrak{a})$ and the contribution to $E$ is

$$
\ll \sum_{Q \leq N \mathfrak{q}<2 Q} \sum_{3 \leq j \ll \log x}\left(\frac{x}{N \mathfrak{q}}\right)^{1 / j} \ll x^{1 / 3} Q^{2 / 3} \log x .
$$

For $j=2$, split the inner sum in (6.5) into subsums of ideals lying in a given ideal class $H$, say, $\bmod \mathfrak{f}$. Fixing $\mathfrak{b} \in H^{-1}$, then $\mathfrak{a} \mathfrak{b}=(\lambda)$, for some $\lambda \in \mathfrak{O}$ for all $\mathfrak{a}$ in subsum. So this smaller sum is less than the number of $\lambda \in \mathfrak{O}$ with $N \lambda \ll(x / N \mathfrak{q})^{1 / 2}$ and $\left|\psi\left(\lambda^{2}\right)-\psi_{1}\right|<l$, for some $\psi_{1}$. This latter condition states that $\lambda$ which, for the imaginary quadratic field $k$, are elements of a lattice in the plane, must lie in two cones. Thus the inner sum in $(6.5)$ is $\ll\left((x / N \mathfrak{q})^{1 / 4}+1\right)\left(l(x / N \mathfrak{q})^{1 / 4}+1\right)$ and the contribution to $E$ is $\ll l x^{1 / 2} Q^{1 / 2}+x^{1 / 4} Q^{3 / 4}+Q$. Hence, since $Q<x, E \ll l x^{1 / 2} Q^{1 / 2}+$ $x^{1 / 3} Q^{2 / 3} \log x$.

The inner sum in (6.4) differs from $\Psi_{l}$ in (3.6) of [5] only by the occurrence of $\mathfrak{q}$. The method of [5] replaces $f(t)$ by $\sum_{m=-\infty}^{\infty} a_{m} \exp (2 \pi i m t)$, which gives rise to the Grössencharaktere $\exp (2 \pi i m \psi(\mathfrak{a}))$, while $g(y)$ is replaced by $\int_{c-i \infty}^{c+i \infty} \widehat{g}(s) y^{-s} d s$, where $\widehat{g}$ is the Mellin transform of $g$. It can be shown that both the infinite summation and integration can be cut-off at finite points with arbitrarily small error. The line of integration can then be moved, picking up contributions from the poles of the integrand. In this way we obtain

$$
\begin{aligned}
& \frac{a_{0} \widehat{g}(1)}{h(\mathfrak{f}) N \mathfrak{q}} \\
- & \frac{1}{h(\mathfrak{f})} \sum_{\chi} \chi(I) \sum_{|m|<W} a_{m} e^{-2 \pi i m \psi_{0}} \sum_{\left|\gamma_{m \chi}\right|<T} \widehat{g}\left(\varrho_{m \chi}\right) \frac{\chi \lambda^{m}(\mathfrak{q})}{N \mathfrak{q}^{\varrho_{m}}}+O(\log x) .
\end{aligned}
$$

Here $\chi$ denotes a character on $I_{\mathfrak{f}} / P_{\mathfrak{f}}, W=\left[\Delta_{1}^{-1} \log ^{2} x\right]=\left[l^{-1} \log ^{3} x\right], T=$ $\left[\Delta_{2}^{-1} \log ^{3} x\right]=\left[\log ^{4} x\right]$ and $\varrho_{m \chi}=\beta_{m \chi}+i \gamma_{m \chi}$ are the non-trivial zeros of the Hecke $L$-function defined as

$$
L\left(s, \chi \lambda^{m}\right)=\sum_{\mathfrak{a}} \frac{\chi \lambda^{m}(\mathfrak{a})}{N \mathfrak{a}^{s}},
$$

for $\operatorname{Re} s>1$. The error of $\log x$ in (6.6) differs from that in (3.8) in [5] because we use the fact that here $\widehat{g}(\sigma+i t) \ll x^{\sigma}$. 
Substitute the second term from (6.6) into (6.4), bounding the resulting term as

$$
\ll l \sum_{\chi} \sum_{|m|<W} \sum_{\left|\gamma_{m \chi}\right|<T} x^{\beta_{m \chi}}\left|\sum_{Q \leq N \mathfrak{q}<2 Q} \frac{\chi \lambda^{m}(\mathfrak{q})}{N \mathfrak{q}^{\varrho_{m \chi}} \log x / N \mathfrak{q}}\right|,
$$

having used $\left|a_{m}\right| \leq a_{0} \ll l$ for all $m$. By partial summation, this is

$$
\ll l x \max _{\beta \geq 1 / 2} x^{\beta-1} \sum_{\chi} \sum_{\substack{|m|<W,\left|\gamma_{m \chi}\right|<T \\ \beta_{m \chi}>\beta}}\left|S\left(Q^{\prime}, \chi \lambda^{m}, \varrho_{m \chi}\right)\right|
$$

for some $Q<Q^{\prime} \leq 2 Q$, where

$$
S\left(Q^{\prime}, \chi \lambda^{m}, s\right)=\sum_{Q<N \mathfrak{q} \leq Q^{\prime}} \chi \lambda^{m}(\mathfrak{q}) N \mathfrak{q}^{-s} .
$$

For $1 / 2 \leq \sigma \leq 1$, define $N(\sigma, W, T)$ to be the number of non-trivial zeros $\varrho_{m \chi}$ of all $L\left(s, \lambda^{m} \chi\right)$ where $|m| \leq W$ and $\chi$ is a character on $I_{\mathfrak{f}} / P_{\mathfrak{f}}$, with $\beta_{m \chi} \geq \sigma$ and $\left|\gamma_{m \chi}\right| \leq T$. Bounds for this counting function are given in [3] but those results are optimal when $W$ and $T$ are of the same magnitude. Because of the geometry of the region in which we are looking for prime ideals in Theorem 2, the $W$ and $T$ in (6.6) are not of the same magnitude. So we quote instead Theorem 3 of [26] as

\section{LEMMA 5.}

$$
N(\sigma, W, T) \ll_{\mathfrak{f}} T W^{2(1-\sigma)}\left(W^{2}+T^{2}\right)^{2(1-\sigma) / 3} \log ^{15} W T,
$$

or, as appropriate for us,

$$
N\left(\sigma, W, \log ^{4} x\right) \ll_{\mathfrak{f}} W^{10(1-\sigma) / 3} \log ^{19} x .
$$

In fact, $(6.8)$ is only given in $[26]$ for $k=\mathbb{Q}(i)$ and $\mathfrak{f}=(1)$. Below I will sketch how (6.8) can be proven in general. The exponent 10/3 in (6.9) is better than the $24 / 5+\varepsilon$ implied by Corollary 8.2 of [3]. The improvement arises from the mean value result

\section{LEMMA 6.}

$$
\sum_{\chi} \sum_{|m|<W}\left|\sum_{N \mathfrak{a}<X} C(\mathfrak{a}) \chi \lambda^{m}(\mathfrak{a})\right|^{2} \ll_{\mathfrak{f}}(W+X) \sum_{N \mathfrak{a}<X}^{*}\left|C_{1}(\mathfrak{a})\right|^{2},
$$

where $C_{1}(\mathfrak{a})=\sum\{C(\mathfrak{b}): N \mathfrak{b}<X, \psi(\mathfrak{b})=\psi(\mathfrak{a}), \mathfrak{b} \in H(\mathfrak{a})\}$. The sums are over integral ideals, $\{C(\mathfrak{a})\}$ is a set of complex numbers, $\sum^{*}$ denotes that the sum is over integral ideals $\mathfrak{a}$ that cannot be written as $m \mathfrak{a}^{\prime}$ with $m \in \mathbb{Z}$ and $\mathfrak{a}^{\prime}$ an integral ideal, and $H(\mathfrak{a})$ is the element of $I_{\mathfrak{f}} / P_{\mathfrak{f}}$ containing $\mathfrak{a}$.

Proof of Lemma 6. This is Theorem C of [26], but for ease of reference I will sketch the proof. The characters in the left-hand side of 
(6.10) take a common value for all $\mathfrak{a} \in H, H \in I_{\mathfrak{f}} / P_{\mathfrak{f}}$. Squaring out and using orthogonality of characters, the left-hand side becomes

$$
\begin{aligned}
h(\mathfrak{f}) \sum_{H \in I_{\mathfrak{f}} / P_{\mathfrak{f}}} \sum_{|m|<W}\left|\sum_{\substack{N \mathfrak{a}<X \\
\mathfrak{a} \in H}} C(\mathfrak{a}) e^{2 \pi i m \psi(\mathfrak{a})}\right|^{2} \\
=h(\mathfrak{f}) \sum_{H \in I_{\mathfrak{f}} / P_{\mathfrak{f}}} \sum_{|m|<W}\left|\sum_{\substack{N \mathfrak{a}<X \\
\mathfrak{a} \in H}}^{*} C_{1}(\mathfrak{a}) e^{2 \pi i m \psi(\mathfrak{a})}\right|^{2},
\end{aligned}
$$

having replaced $\lambda(\mathfrak{a})$ by $e^{2 \pi i \psi(\mathfrak{a})}$. If $\mathfrak{a}_{1}, \mathfrak{a}_{2} \in H$ occur in $\sum^{*}$ then $\psi\left(\mathfrak{a}_{1}\right)-$ $\psi\left(\mathfrak{a}_{2}\right) \neq 0$. Let $\mathfrak{c} \in H^{-1}$; then

$$
\begin{aligned}
\psi\left(\mathfrak{a}_{1}\right)-\psi\left(\mathfrak{a}_{2}\right) & =\psi\left(\mathfrak{a}_{1} \mathfrak{c}\right)-\psi\left(\mathfrak{a}_{2} \mathfrak{c}\right)=\psi\left(\xi_{1}\right)-\psi\left(\xi_{2}\right) \\
& =\omega(\mathfrak{f})\left\{\arg \xi_{1}-\arg \xi_{2}\right\}=\omega(\mathfrak{f}) \arg \xi_{1} \bar{\xi}_{2},
\end{aligned}
$$

where $\xi_{1}, \xi_{2} \in \mathfrak{c}$ and $\xi_{1}, \xi_{2} \equiv 1 \bmod \mathfrak{f}$. But $\xi_{1} \bar{\xi}_{2} \in \mathfrak{O}_{k}$ and in general if $\alpha \in$ $\mathfrak{O}_{k}$ then $\arg \alpha=0$ if and only if $\alpha \in \mathbb{Z}$, and if $\alpha \notin \mathbb{Z},|\arg \alpha| \gg_{k} 1 / N \alpha^{1 / 2}$. So if $\psi\left(\mathfrak{a}_{1}\right) \neq \psi\left(\mathfrak{a}_{2}\right)$, we have

$$
\left|\psi\left(\mathfrak{a}_{1}\right)-\psi\left(\mathfrak{a}_{2}\right)\right| \gg_{k} 1 /\left(N \xi_{1} \xi_{2}\right)^{1 / 2}=1 /\left(N \mathfrak{a}_{1} \mathfrak{a}_{2}\right)^{1 / 2} N \mathfrak{c} \geq 1 / X N \mathfrak{c} .
$$

But $\mathfrak{c}$ can be chosen such that $N \mathfrak{c} \ll_{\mathfrak{f}} 1$, so $\left|\psi\left(\mathfrak{a}_{1}\right)-\psi\left(\mathfrak{a}_{2}\right)\right| \geq c(\mathfrak{f}) / X$ for some $c(\mathfrak{f})>0$.

Apply to (6.11) the dual of the large sieve, namely

$$
\sum_{|n|<N}\left|\sum_{r \in \mathbb{R}} a_{r} e^{2 \pi i n x_{r}}\right|^{2} \ll\left(N+\delta^{-1}\right) \sum_{r \in \mathbb{R}}\left|a_{r}\right|^{2},
$$

where the $x_{i}$ are real numbers distinct modulo 1 , and $\delta=\min _{r \neq s} \| x_{r}-$ $x_{s} \|$, where $\|x\|$ denotes the distance from $x$ to the nearest integer. In our application $\delta=c(\mathfrak{f}) / X$, thus $(6.11)$ is

$$
\ll_{\mathfrak{f}}(W+X) \sum_{H} \sum_{\substack{N \mathfrak{a}<X \\ \mathfrak{a} \in H}}^{*}\left|C_{1}(\mathfrak{a})\right|^{2},
$$

as required.

Proof of Lemma 5. Let $T \geq 2, \delta>0$ and assume for each $m, \chi$ there is given $T_{m \chi}=\left\{t_{m \chi i}\right\} \subseteq[-T+\delta / 2, T-\delta / 2]$, a set of points wellspaced by $\delta$; that is, $\left|t_{m \chi i}-t_{m \chi j}\right| \geq \delta$ when $i \neq j$. Then from Lemma 6 and Lemma 1.4 of Gallagher [6] we deduce

$$
\begin{aligned}
\sum_{\chi} \sum_{|m|<W} \sum_{t_{m \chi} \in T_{m \chi}} \mid & \sum_{N \mathfrak{a}<X} C(\mathfrak{a}) \chi \lambda^{m}(\mathfrak{a}) N \mathfrak{a}^{i t_{m \chi}} \mid \\
& \ll_{\mathfrak{f}} T\left(\delta^{-1}+\log X\right)(W+X) \sum_{N \mathfrak{a}<X}^{*}\left|C_{1}(\mathfrak{a})\right|^{2} .
\end{aligned}
$$


For the method of proof of the zero density result (6.8) we quote from [3]. First, note that with $m, \chi$ fixed, the number of non-trivial zeros $\varrho_{m \chi}$ with $T<\left|\gamma_{m \chi}\right|<T+1$ is $\ll \log |m| T$, ([22], Lemma 12), so $N(\sigma, W, T) \ll$ $N^{*}(\sigma, W, T) \log ^{2} W T$, where, for fixed $m, \chi$, the zeros in $N^{*}(\sigma, W, T)$ are well-spaced by $\gg \log W T$. For zeros counted in $N^{*}(\sigma, W, T)$ we have either

$$
\left|\sum_{U \leq N \mathfrak{a}<2 U} A(\mathfrak{a}) \chi \lambda^{m}(\mathfrak{a}) N \mathfrak{a}^{-\varrho_{m \chi}}\right| \gg(\log W T)^{-1},
$$

or for each $\varrho_{m \chi}$ there exists $t_{m \chi}$ with $\left|t_{m \chi}-\gamma_{m \chi}\right| \ll \log W T$ for which

$$
\begin{aligned}
\mid L\left(1 / 2+i t_{m \chi}, \chi \lambda^{m}\right) \sum_{N \mathfrak{a}<X} \mu(\mathfrak{a}) \chi \lambda^{m}(\mathfrak{a}) & N \mathfrak{a}^{-1 / 2-i t_{m \chi}} \mid \\
& \gg Y^{\beta_{m \chi}-1 / 2}(\sigma-1 / 2) .
\end{aligned}
$$

Here $X \leq Y$ are to be chosen, $X<U \ll Y \log W T, \mu$ is the Möbius function and $|A(\mathfrak{a})| \leq \sigma(\mathfrak{a})$, the number of divisors of $\mathfrak{a}$.

In the first case (6.14), we replace $\beta_{m \chi}$ with $\sigma$ using partial summation, square the result and use (6.13). If $\mathfrak{a}$ is counted in $\sum^{*}$ and $\psi(\mathfrak{b})=\psi(\mathfrak{a})$ then, by $(6.12), \omega(\mathfrak{f}) \arg \xi_{1} / \xi_{2}=0$ for some $\left(\xi_{1}\right)=\mathfrak{b} \mathfrak{c},\left(\xi_{2}\right)=\mathfrak{a c}$. That is, there exist $m, n \in \mathbb{Z}$ and a unit $\varepsilon \equiv 1 \bmod \mathfrak{f}$ such that $\xi_{1} / \xi_{2}=\varepsilon m / n$, or equivalently $(n) \mathfrak{b}=(m) \mathfrak{a}$. If $(n) \neq \mathfrak{O}_{k}$ then $(n) \mid \mathfrak{a}$ because $(m, n)=1$, and so $\mathfrak{a}$ cannot be counted in $\sum^{*}$. Contradiction, so $(n)=\mathfrak{O}_{k}$ and $\mathfrak{b}=(m) \mathfrak{a}$. Thus

$$
\begin{gathered}
C_{1}(\mathfrak{a})=\sum_{U<N((m) \mathfrak{a})<2 U} \frac{A((m) \mathfrak{a})}{N((m) \mathfrak{a})^{\sigma}} \leq \frac{\sigma(\mathfrak{a})}{N \mathfrak{a}^{\sigma}} \sum_{U_{1}<m<U_{2}} \frac{\sigma^{2}(m)}{m^{2 \sigma}} \\
\ll \frac{\sigma(\mathfrak{a})}{N \mathfrak{a}^{\sigma}}\left(\frac{U}{N \mathfrak{a}}\right)^{(1-2 \sigma) / 2} \log ^{3} U, \\
\left(U_{1}=(U / N \mathfrak{a})^{1 / 2}, U_{2}=(2 U / N \mathfrak{a})^{1 / 2}\right), \text { and so } \\
\sum_{N \mathfrak{a} \leq X}^{*}\left|C_{1}(\mathfrak{a})\right|^{2} \ll U^{1-2 \sigma} \log ^{6} U \sum_{N \mathfrak{a} \leq u} \frac{\sigma^{2}(\mathfrak{a})}{N \mathfrak{a}} \ll U^{1-2 \sigma} \log ^{10} U .
\end{gathered}
$$

Hence the number of $\varrho_{m \chi}$ counted in $N^{*}\left(\sigma_{x}, W, T\right)$ for which (6.14) holds is

$$
\ll T\left(W X^{1-2 \sigma}+Y^{2-2 \sigma}\right) \log ^{11} W T .
$$

For the second case (6.15) note first that the final result is trivial for $1 / 2 \leq \sigma \leq 1 / 2+1 / \log W T$, so we can assume $\sigma \geq 1 / 2+1 / \log W T$. Apply the order result for Hecke $L$-functions in imaginary quadratic fields due to Kaufman [18]:

$$
L\left(1 / 2+i t, \chi \lambda^{m}\right) \ll\left(m^{2}+t^{2}\right)^{1 / 6} \log ^{4}\left(m^{2}+t^{2}\right) .
$$


Again square the result and apply (6.13) to see that the number of $\varrho_{m \chi}$ for which (6.15) holds is

$$
\ll T Y^{1-2 \sigma}\left(W^{2}+T^{2}\right)^{1 / 3}(W+X) \log ^{13} W T .
$$

If we choose $X=W$ and $Y=W(W+T)^{2 / 3}$ then (6.8) follows.

We return to (6.7) and ensure the $\gamma_{m \chi}$ are well-spaced by splitting $[-T, T]$ into a union of intervals $[n, n+1)$ and for each $n$, choosing the zero $\widehat{\varrho}_{m \chi}$ with $n \leq \widehat{\gamma}_{m \chi}<n+1$ and $\left|S\left(Q^{\prime}, \chi \lambda^{m}, \widehat{\varrho}_{m \chi}\right)\right|$ maximum. Splitting into two cases $n$ even or odd, we see that in each case $\left|\widehat{\gamma}_{m \chi}-\widehat{\gamma}_{m \chi}^{\prime}\right|>1$. Then

$$
\begin{aligned}
& \sum_{\chi} \sum_{\substack{|m|<W \\
\beta_{m \chi}>\beta}} \sum_{\left|\gamma_{m \chi}\right|<T}\left|S\left(Q^{\prime}, \chi \lambda^{m}, \varrho_{m \chi}\right)\right| \\
& \ll \log ^{2} W T \sum_{\chi} \sum_{\substack{|m|<W \\
\hat{\beta}_{m \chi}>\beta}} \sum_{\left|\hat{\gamma}_{m \chi}\right|<T}\left|S\left(Q^{\prime}, \chi \lambda^{m}, \widehat{\varrho}_{m \chi}\right)\right| \\
& \ll \log ^{2} W T \cdot N^{1 / 2}(\beta, W, T)\left(\sum_{\chi} \sum_{|m| \leq W} \sum_{\substack{\left|\hat{\gamma}_{m \chi}\right|<T \\
\hat{\beta}_{m \chi}>\beta}}\left|S\left(Q^{\prime}, \chi \lambda^{m}, \widehat{\varrho}_{m \chi}\right)\right|^{2}\right)^{1 / 2} \\
& \ll W^{5(1-\beta) / 3}\left\{Q^{1-2 \beta}(W+Q)\right\}^{1 / 2} \log ^{11 / 2} W T
\end{aligned}
$$

(using (6.9) and (6.10)),

$$
\ll W^{5(1-\beta) / 3} Q^{1-\beta} \log ^{11 / 2} W T,
$$

for $Q \geq W$. If we substitute this bound into (6.7) and demand that, given $\varepsilon>0, Q<x^{1-\varepsilon} W^{-5 / 3}$ then $(6.7)$ is $\ll l x \log ^{11 / 2} W T \max x^{-\varepsilon(1-\beta)}$. We use the zero-free region as stated in

Lemma 7. For all $\chi \bmod \mathfrak{f}$ and all $|m| \leq W$, the Hecke L-functions $L\left(s, \chi \lambda^{m}\right)$ have no zero in the region

$$
\sigma \geq 1-c_{1}(\mathfrak{f})(\log W T)^{-\alpha}, \quad|t|<T
$$

when $W^{2}+T^{2} \geq c_{2}(\mathfrak{f})$ for constants $c_{1}$ and $c_{2}$ and some $0<\alpha<1$.

See either [21], Lemma 13 or [4], Theorem 2 for details of the proof. The bound (6.7) is thus seen to be

$$
\ll l x^{1-c_{1}(\mathfrak{f}) \varepsilon / \log ^{\alpha} x} \log ^{11 / 2} W T \ll l x / \log ^{2} x
$$

for $x>x_{0}(\varepsilon, \mathfrak{f})$.

If we substitute the first term from (6.6) into (6.4) we obtain

$$
\frac{2 l x}{h(\mathfrak{f})}\left(1+O\left(\frac{1}{\log x}\right)\right) \sum_{Q<N \mathfrak{q}<2 Q} \frac{1}{N \mathfrak{q} \log x / N \mathfrak{q}}
$$


having used $a_{0}=2 l+O\left(\Delta_{1}\right)=2 l(1+O(1 / \log x))$ and $\widehat{g}(1)=x+O\left(\Delta_{2} x\right)=$ $x(1+O(1 / \log x))$. By the prime number theorem for prime ideals the sum in (6.18) is $\gg 1 / \log x$ and so the term above dominates (6.17).

Finally, the error in (6.6) contributes essentially nothing, while in the range $Q<x^{1-\varepsilon} W^{-5 / 3}$, the error $E$ in $(6.4)$ is $\ll l^{11 / 6} x^{1-\varepsilon / 2}+l^{10 / 9} x^{1-2 \varepsilon / 3} \ll$ $l x / \log ^{2} x$. Hence, on evaluating the sum in (6.18) using partial summation, we have

Proposition 8. Let $\varepsilon>0$ be given. For $W \leq Q \leq x^{1-\varepsilon} W^{-5 / 3}$ we have

$$
S(Q)=\frac{2 l x}{h(\mathfrak{f}) \log x}\left(1+O\left(\frac{1}{\log x}\right)\right) \int_{Q_{1}}^{Q_{2}} \frac{d t}{t(1-t)},
$$

for $x>x_{0}(\varepsilon, \mathfrak{f})$, where $Q_{1}=\log Q / \log x$ and $Q_{2}=\log 2 Q / \log x$.

The above method gives an asymptotic result for $\sum \theta(\mathfrak{p})$. From (6.3) this would be the case $Q=1$. The only difference in the proof would occur at (6.7); the summand is now 1 and Lemma 5 can be used directly obtaining a bound $\ll l x / \log ^{2} x$ for $(6.7)$ if $W^{10 / 3} / x<x^{-\varepsilon}$. Since $W=l^{-1} \log ^{3} x$ this condition can be given as $l>x^{-3(1-\varepsilon) / 10}$. From (6.1), we have that $\sum \theta(\mathfrak{p})$ is a lower bound for an unweighted sum over prime ideals. As in $[3]$ an upper bound, $\sum \widetilde{\theta}(\mathfrak{p})$ say, can be given for the unweighted sum, with the properties of $\widetilde{\theta}(\mathfrak{p})$ so similar to those of $\theta(\mathfrak{p})$ that the same asymptotic results hold for $\sum \widetilde{\theta}(\mathfrak{p})$. Hence

Theorem 3. Let $\varepsilon>0$ be given. For $1 / 2>l>x^{-3(1-\varepsilon) / 10}$ we have

$$
\sum_{\substack{\mathfrak{p} \in I \\\left|\psi(\mathfrak{p})-\psi_{0}\right|<l \\ x<N \mathfrak{p}<2 x}} 1=\frac{2 l x}{h(\mathfrak{f}) \log x}\left(1+O\left(\frac{1}{\log x}\right)\right),
$$

for all $\psi_{0} \in[0,1), x>x_{0}(\varepsilon, \mathfrak{f})$.

This is Theorem 2 of [26] generalized to arbitrary quadratic imaginary fields.

7. Application of sieve results. We can use Proposition 8 to estimate $\sum_{z<N \mathfrak{q}<Z} S\left(\mathcal{A}_{\mathfrak{q}}, \mathfrak{q}\right)$ for $z=W, Z=x^{1-\varepsilon} W^{-5 / 3}$ as long as $z \geq x^{1 / 3}$. For the remaining terms in (6.2) we apply Lemma 3. Consider $\sum_{\mathfrak{d} \mid \mathfrak{a}} \theta(\mathfrak{a})$, for $\mathfrak{d}$ an integral ideal. If $(\mathfrak{d}, \mathfrak{f}) \neq(1)$, there can exist no ideal $\mathfrak{a}$ in this sum such that $\mathfrak{a} \in I \in I_{\mathfrak{f}} / P_{\mathfrak{f}}$, hence the sum is zero. If $(\mathfrak{d}, \mathfrak{f})=(1)$, then by the argument preceding (6.6),

$$
\sum_{\mathfrak{d} \mid \mathfrak{a}} \theta(\mathfrak{a})=\frac{1}{h(\mathfrak{f})} \sum_{\chi} \bar{\chi}(I) \sum_{m=-\infty}^{\infty} a_{m} \frac{1}{2 \pi i} \int_{c-i \infty}^{c+i \infty} \frac{\widehat{g}(s) \chi \lambda^{m}(\mathfrak{d})}{N \mathfrak{d}^{s}} L\left(s, \chi \lambda^{m}\right) d s
$$


for any $c>1$. The integrand has a pole at $s=1$, when $m=0, \chi=\chi_{0}$, of residue $a_{0} \widehat{g}(1) \operatorname{Res}_{s=1} L\left(s, \chi_{0}\right)(h(\mathfrak{f}) N \mathfrak{d})^{-1}$. In view of $(2.5)$, define

$$
X=a_{0} \widehat{g}(1) \operatorname{Res}_{s=1} L\left(s, \chi_{0}\right) h^{-1}(\mathfrak{f})
$$

and

$$
\omega(\mathfrak{d})= \begin{cases}1 & \text { if }(\mathfrak{d}, \mathfrak{f})=(1) \\ 0 & \text { if }(\mathfrak{d}, \mathfrak{f}) \neq(1) .\end{cases}
$$

From (2.3) of [29] we have Mertens's Theorem for any number field $K$, namely

$$
W(z):=\prod_{N \mathfrak{p}<z}\left(1-\frac{1}{N \mathfrak{p}}\right)=\frac{e^{-\gamma}}{a_{K}} \frac{1}{\log z}+O\left(\frac{1}{\log ^{2} z}\right),
$$

where $a_{K}$ is the residue of $\zeta_{K}(s)$, the Dedekind zeta-function, at $s=1$ and $\gamma$ denotes Euler's constant. Also,

$$
\prod_{\mathfrak{h} \leq K \mathfrak{p}<<_{K} \mathfrak{j}}\left(1-\frac{\omega(\mathfrak{p})}{N \mathfrak{p}}\right)^{-1} \leq \frac{W(N \mathfrak{h})}{W(N \mathfrak{j})}\left(1+O\left(\frac{1}{N \mathfrak{h}}\right)\right),
$$

where the $O(1 / N \mathfrak{h})$ term accounts for the prime ideals that are lost or gained when $\mathfrak{h} \leq_{K} \mathfrak{p}<_{K} \mathfrak{j}$ is replaced by $N \mathfrak{h} \leq N \mathfrak{p}<N \mathfrak{j}$. But now (3.3) follows immediately from (7.2) and (7.3), while (3.10) follows from the prime ideal theorem.

The application of Lemma 3 will be exactly as in [8]. Consider $V(z) X$ and for simplicity assume $z>N \mathfrak{f}$. Then since

$\operatorname{Res}_{s=1} L\left(s, \chi_{0}\right)=\prod_{\mathfrak{p} \mid \mathfrak{f}}(1-1 / N \mathfrak{p}) \operatorname{Res}_{s=1} \zeta_{k}(s) \quad$ and $\quad V(z)=\prod_{\mathfrak{p} \mid \mathfrak{f}}(1-1 / N \mathfrak{p})^{-1} W(z)$

we deduce from (7.2) that

$$
V(z) X=a_{0} \widehat{g}(1)(h(\mathfrak{f}) \log z)^{-1}(1+O(1 / \log z)) .
$$

Thus for any $\varepsilon>0$ and with $D>z^{2}$ to be chosen,

$$
S(\mathcal{A}, z) \geq \frac{a_{0} \widehat{g}(1)}{h(\mathfrak{f}) \log z}\left\{f\left(\frac{\log D}{\log z}\right)-c \varepsilon+O\left(\frac{1}{(\log D)^{1 / 3}}\right)\right\}-R^{-}(\mathcal{A}, D)
$$

For the remaining term in (6.2) split the range of the prime $\mathfrak{q}, Z<N \mathfrak{q}<$ $x^{1 / 2}$, into subsums with $Q \leq N \mathfrak{q}<2 Q$. In each such subsum $N \mathfrak{q}>$ $(D / 2 Q)^{1 / 3}$ and so using the observation that $S(\mathcal{A}, \mathfrak{p}) \leq S(\mathcal{A}, w)$ if $N \mathfrak{p}>w$ we have

$$
\sum_{Z<N \mathfrak{p}<x^{1 / 2}} S\left(\mathcal{A}_{\mathfrak{p}}, \mathfrak{p}\right) \leq \sum_{Q} \frac{1}{\log Q} \sum_{\substack{Q<N \mathfrak{a}<2 Q \\ Z<N \mathfrak{a}<x^{1 / 2}}} \Lambda(\mathfrak{a}) S\left(\mathcal{A}_{\mathfrak{a}},(D / 2 Q)^{1 / 3}\right) .
$$


By Lemma 3, the inner sum on the right-hand side is

$$
\begin{aligned}
& \leq \frac{a_{0} \widehat{g}(1)}{h(\mathfrak{f})} \sum_{\substack{Q \leq N \mathfrak{a}<2 Q \\
Z<N \mathfrak{a}<x^{1 / 2}}} \frac{\Lambda(\mathfrak{a})}{N \mathfrak{a} \log \left((D / 2 Q)^{1 / 3}\right)} \\
& \quad \times\left\{F(3)+c \varepsilon+O\left(\frac{1}{(\log (D / 2 Q))^{1 / 3}}\right)\right\} \\
& +\sum_{\substack{Q \leq N \mathfrak{a}<2 Q \\
Z<N \mathfrak{a}<x^{1 / 2}}} \Lambda(\mathfrak{a}) R^{+}\left(\mathcal{A}_{\mathfrak{a}}, D / 2 Q\right) .
\end{aligned}
$$

To the sum over errors we apply the method of Vaughan [31]. This method has been used in number fields by Hinz [11]. He derives the required identity by following the original idea of Vaughan, giving an identity for $-\zeta_{K}^{\prime}(s) / \zeta_{K}(s)$ and then equating coefficients. Yet it is possible to have $\sum A(\mathfrak{a}) N \mathfrak{a}^{-s}=\sum B(\mathfrak{a}) N \mathfrak{a}^{-s}$ for all $s \in \mathbb{C}$, where both sums are over integral ideals from $K$, without $A(\mathfrak{a})=B(\mathfrak{a})$ for all $\mathfrak{a}$. The most that can be deduced is that

$$
\sum_{N \mathfrak{a}=n} A(\mathfrak{a})=\sum_{N \mathfrak{a}=n} B(\mathfrak{a}), \quad \text { for all } n \in \mathbb{Z} .
$$

Here we quote from [9], defining sums $\Sigma_{j}(Q, u)(0 \leq j \leq 5)$ by

$$
\Sigma_{j}(Q, u)=\sum_{\mathfrak{a}, \mathfrak{b}, \mathfrak{c}} \Lambda(\mathfrak{a}) \mu(\mathfrak{b}) R^{+}\left(\mathcal{A}_{\mathfrak{a} \mathfrak{b} \mathfrak{c}}, D / 2 Q\right),
$$

where $u$ is a real number, $\mathfrak{a}, \mathfrak{b}$ and $\mathfrak{c}$ run through integral ideals of $K$ and the range of summation is $Q \leq N \mathfrak{a} \mathfrak{b} \mathfrak{c}<2 Q, Z \leq N \mathfrak{a} \mathfrak{b} \mathfrak{c}<x^{1 / 2}$ and if

$$
\begin{array}{ll}
j=0, & N \mathfrak{b} \mathfrak{c} \leq u, \\
j=1, & N \mathfrak{b} \leq u, \\
j=2, & N \mathfrak{a} \mathfrak{b} \leq u, \\
j=3, & N \mathfrak{a} \mathfrak{b} \geq u, \quad N \mathfrak{a} \leq u, \quad N \mathfrak{b} \leq u, \\
j=4, & N \mathfrak{a}>u, \quad N \mathfrak{b} \mathfrak{c}>u, \quad N \mathfrak{b} \leq u, \\
j=5, & N \mathfrak{b} \mathfrak{c} \leq u, \quad N \mathfrak{a} \leq u .
\end{array}
$$

Then Vaughan's identity is

$$
\Sigma_{0}(Q, u)+\Sigma_{2}(Q, u)+\Sigma_{3}(Q, u)+\Sigma_{4}(Q, u)=\Sigma_{1}(Q, u)+\Sigma_{5}(Q, u) .
$$

Here $u$ is to be chosen. If we assume that $1 \leq u \leq Q^{1 / 2}$ then, since the range of summation is empty, $\Sigma_{5}(Q, u)=0$. On writing $\mathfrak{d}=\mathfrak{b} \mathfrak{e}$, we see that 


$$
\begin{aligned}
\Sigma_{0}(Q, u) & =\sum_{Q \leq N \mathfrak{a} \mathfrak{d}<2 Q}^{\prime} \Lambda(\mathfrak{a})\left(\sum_{\mathfrak{b} \mid \mathfrak{d}} \mu(\mathfrak{b})\right) R^{+}\left(\mathcal{A}_{\mathfrak{a} \mathfrak{d}}, D / 2 Q\right) \\
& =\sum_{Q \leq N \mathfrak{a}<2 Q}^{\prime} \Lambda(\mathfrak{a}) R^{+}\left(\mathcal{A}_{\mathfrak{a}}, D / 2 Q\right),
\end{aligned}
$$

which is what we are estimating. Here the prime denotes $Z \leq N \mathfrak{a d} \leq x^{1 / 2}$. We deal with the other sums in the same way of combining variables.

In $\Sigma_{3}$ write $\mathfrak{f}=\mathfrak{a} \mathfrak{b}, \mathfrak{h}=\mathfrak{c}$ while in $\Sigma_{4}, \mathfrak{f}=\mathfrak{a}$ and $\mathfrak{h}=\mathfrak{b} \mathfrak{c}$. In $\Sigma_{3}$ we have $u \leq N \mathfrak{f}<u^{2}$ which is $<2 Q / u$ if $u<(2 Q)^{1 / 3}$ as we now assume. Thus, in both cases, the sums have the form

$$
\Sigma_{\mathrm{I}}(Q)=\sum_{\substack{Q<N \mathfrak{h}<2 Q \\ u<N \mathfrak{f}<2 Q / u}} A(\mathfrak{f}) B(\mathfrak{h}) R^{+}\left(\mathcal{A}_{\mathfrak{f h}}, D / 2 Q\right),
$$

where from $\Sigma_{3}$,

$$
A(\mathfrak{f})=\sum_{\substack{\mathfrak{a} \mathfrak{b}=\mathfrak{f} \\ N \mathfrak{a} \leq u \\ N \mathfrak{b} \leq u}} \Lambda(\mathfrak{a}) \mu(\mathfrak{b}) \ll \log N \mathfrak{f}, \quad B(\mathfrak{h})=1
$$

and from $\Sigma_{4}$,

$$
A(\mathfrak{f})=\Lambda(\mathfrak{f}) \ll \log N \mathfrak{f} \quad \text { and } \quad B(\mathfrak{h})=\sum_{\substack{\mathfrak{c} \mid \mathfrak{h} \\ N \mathfrak{c} \leq u}} \mu(\mathfrak{c}) \ll \sigma(\mathfrak{h}) .
$$

In $\Sigma_{1}$ write $\mathfrak{f}=\mathfrak{b}, \mathfrak{h}=\mathfrak{a} \mathfrak{c}$ while in $\Sigma_{2}, \mathfrak{f}=\mathfrak{a} \mathfrak{b}$ and $\mathfrak{h}=\mathfrak{c}$. Then both sums are examples of

$$
\Sigma_{\mathrm{II}}(Q)=\sum_{\substack{Q \leq N \mathfrak{f h}<2 Q \\ N \mathfrak{f} \leq u}} A(\mathfrak{f}) B(\mathfrak{h}) R^{+}\left(\mathcal{A}_{\mathfrak{f h}}, D / 2 Q\right),
$$

and from $\Sigma_{1}$,

$$
A(\mathfrak{f})=\mu(\mathfrak{f}) \ll 1, \quad B(\mathfrak{h})=\sum_{\mathfrak{a} \mathfrak{c}=\mathfrak{h}} \Lambda(\mathfrak{a})=\log N \mathfrak{h}
$$

and from $\Sigma_{2}$,

$$
A(\mathfrak{f})=\sum_{\mathfrak{a} \mathfrak{b}=\mathfrak{f}} \Lambda(\mathfrak{a}) \mu(\mathfrak{b}) \ll \log N \mathfrak{f} \quad \text { and } \quad B(\mathfrak{h})=1 .
$$

Thus to estimate the error in (7.5) it suffices to bound sums of the form $\Sigma_{\mathrm{I}}, \Sigma_{\mathrm{II}}$ with any coefficients satisfying the bounds above.

Looking first at $\Sigma_{\mathrm{I}}$ we split the sum in $O(\log Q)$ subsums by restricting the variables to ranges such as $F \leq N \mathfrak{f}<2 F, H \leq N \mathfrak{h}<2 H$ where $F \geq u$, $H \geq u / 2$ and $F H<2 Q$. For each such subsum the condition $Q \leq N \mathfrak{f h}<2 Q$ 
is removed by using Perron's Theorem ([30], Lemma 3.12), which allows us to write the subsum as

$$
\begin{aligned}
& \frac{1}{2 \pi i} \int_{-i T}^{i T} \frac{(2 Q)^{u}-Q^{u}}{u} \\
& \times \sum_{\substack{F \leq N \mathfrak{f}<2 F \\
H \leq N \mathfrak{h}<2 H}} A(\mathfrak{f}) N \mathfrak{f}^{-u} B(\mathfrak{h}) N \mathfrak{h}^{-u} R^{+}\left(\mathcal{A}_{\mathfrak{f h}}, D / 2 Q\right) d u+O(1) \\
& \ll \log T\left|\sum_{\substack{F \leq N \mathfrak{f}<2 F \\
H \leq N \mathfrak{h}<2 H}} \widetilde{A}(\mathfrak{f}) \widetilde{B}(\mathfrak{h}) R^{+}\left(\mathcal{A}_{\mathfrak{f h}}, D / 2 Q\right)\right|
\end{aligned}
$$

Here $T$ is a sufficiently large power of $x$ and $\widetilde{A}(\mathfrak{f})=A(\mathfrak{f}) N f^{-i t}, \widetilde{B}(\mathfrak{h})=$ $B(\mathfrak{h}) N h^{-i t}$ for some $-T \leq t \leq T$. Now use (4.1) within (7.6), expressing $R^{+}\left(\mathcal{A}_{\mathfrak{f h}}, D / 2 Q\right)$ as a sum of

$$
\sum_{N \mathfrak{e}<E} \sum_{N \mathfrak{g}<G} a(\mathfrak{e}) b(\mathfrak{g}) r\left(\mathcal{A}_{\mathfrak{f h}}, \mathfrak{e} \mathfrak{g}\right)
$$

with $|a(\mathfrak{e})| \leq 1,|b(\mathfrak{g})| \leq 1$ and $E G=D / 2 Q$. Yet $r\left(\mathcal{A}_{\mathfrak{f h}}, \mathfrak{e g}\right)=r(\mathcal{A}, \mathfrak{e f g} \mathfrak{g})$ and so on substituting each sum (7.7) into (7.6) we can combine variables by defining $\mathfrak{a}=\mathfrak{f} \mathfrak{e}$ and $\mathfrak{b}=\mathfrak{g h}$. The lengths of the resulting bilinear sums are $N \mathfrak{a}<A, N \mathfrak{b}<B$ with $A>F, B>H$ and $A B=D$, while the coefficients are essentially divisor functions and so are $\ll_{\varepsilon_{1}} x^{\varepsilon_{1}}$ for any $\varepsilon_{1}>0$. There are $\ll_{\varepsilon} 1$ sums of the form $(7.7)$ so, $\Sigma_{\mathrm{I}}(Q) \ll_{\varepsilon, \varepsilon_{1}} x^{\varepsilon_{1}}|R(A, B)|$, where

$$
R(A, B)=\sum_{A / 2<N \mathfrak{a}<A} \sum_{B / 2<N \mathfrak{b}<B} A(\mathfrak{a}) B(\mathfrak{b}) r(\mathcal{A}, \mathfrak{a} \mathfrak{b}),
$$

for some $A B \leq D, A, B<2 Q / u$ and coefficients satisfying $|A(\mathfrak{a})| \leq 1$, $|B(\mathfrak{b})| \leq 1$. Thus for both $\Sigma_{\mathrm{I}}(Q)$ and the error in the application of the lower bound sieve, (7.4), the following is relevant.

Lemma 9. Let $\eta>0$ be given. Let $x^{7 \eta / 3}<W<x^{3 / 8-5 \eta / 4}, A, B<$ $x^{1-3 \eta} W^{-5 / 3}$ and $A B<x^{1-3 \eta}$. Then

$$
R(A, B) \ll l x^{1-\eta} .
$$

Proof. By definition

$$
r(\mathcal{A}, \mathfrak{a} \mathfrak{b})=\sum_{\mathfrak{a} \mathfrak{b} \mid \mathfrak{c}} \theta(\mathfrak{c})-\omega(\mathfrak{a} \mathfrak{b}) X / N \mathfrak{a} \mathfrak{b},
$$

and in this we use (7.1). As in the proof of (6.6) the properties of $a_{m}$ and $\widehat{g}(s)$ allow us to truncate the sum and integral at $W=l^{-1} \log ^{3} x$ and $T=\log ^{4} x$ respectively. Moving the line of integration to $\operatorname{Re} s=1 / 2$, the contributions on the horizontal lines are arbitrarily small while the pole at 
$s=1$ gives the main term in $r(\mathcal{A}, \mathfrak{a} \mathfrak{b})$. Summing over $\mathfrak{a}$ and $\mathfrak{b}$ gives

$$
\begin{aligned}
R(A, B) & \sim \frac{1}{2 \pi h(\mathfrak{f}) i} \\
\times & \sum_{\chi} \bar{\chi}(I) \sum_{|m|<W} a_{m} \int_{1 / 2-i T}^{1 / 2+i T} \widehat{g}(s) A\left(s, \chi \lambda^{m}\right) B\left(s, \chi \lambda^{m}\right) L\left(s, \chi \lambda^{m}\right) d s,
\end{aligned}
$$

where $\sim$ denotes that the two sides differ by an arbitrarily small amount and $A\left(s, \chi \lambda^{m}\right)=\sum_{A / 2<N \mathfrak{a}<A} A(\mathfrak{a}) \chi \lambda^{m}(\mathfrak{a}) N \mathfrak{a}^{-s}$, similarly for $B\left(s, \chi \lambda^{m}\right)$. Using the order result for $L\left(s, \chi \lambda^{m}\right),(6.16)$, and Cauchy-Schwarz followed by the mean value result, Lemma 6 , we can bound the right-hand side as

$$
\ll l x^{1 / 2} W^{1 / 3}(W+A)^{1 / 2}(W+B)^{1 / 2} \log ^{c} x,
$$

for some $c>0$ having used $g(1 / 2+i t) \ll x^{1 / 2}$. If $A, B<W$ this bound is

$$
\ll l x^{1 / 2} W^{4 / 3} \log ^{c} x \ll l x^{1-5 \eta / 3} \log ^{c} x .
$$

If either $A>W, B<W$ or $A<W, B>W$ the bound is

$$
\ll l x^{1 / 2} W^{5 / 6}\left(x^{1-3 \eta} W^{-5 / 3}\right)^{1 / 2} \log ^{c} x \ll l x^{1-3 \eta / 2} \log ^{c} x .
$$

For the final case $A, B>W$ we deal with the $m=0, \chi=\chi_{0}$ term separately, when

$$
\begin{aligned}
\frac{a_{0}}{2 \pi h(\mathfrak{f}) i} \int_{1 / 2-i T}^{1 / 2+i T} \widehat{g}(s) A\left(s, \chi_{0}\right) B\left(s, \chi_{0}\right) L\left(s, \chi_{0}\right) d s & \ll l x^{1 / 2}(A B)^{1 / 2} \log ^{c} x \\
& \ll l x^{1-3 \eta / 2} \log ^{c} x,
\end{aligned}
$$

having trivially estimated all terms from above. For the remaining terms return to (7.8), writing $\mathfrak{c}=\mathfrak{a b} \mathfrak{d}$ and noting that $\theta(\mathfrak{c})$ is non-zero only if $x / 4 A B<N \mathfrak{d}<2 x / A B$. Thus the $L$-function in (7.9) can be replaced by

$$
N\left(s, \chi \lambda^{m}\right)=\sum_{N<N \mathfrak{a}<8 N} \chi \lambda^{m}(\mathfrak{a}) N \mathfrak{a}^{-s} \quad \text { where } N=x / 4 A B .
$$

From Perron's Theorem we have

$$
\begin{aligned}
N\left(1 / 2+i t, \chi \lambda^{m}\right)= & \frac{1}{2 \pi i} \int_{c-i \tau}^{c+i \tau} \frac{(8 N)^{s}-N^{s}}{s} L\left(s+1 / 2+i t, \chi \lambda^{m}\right) d s \\
& +O_{\eta}\left(\frac{N^{1 / 2+\eta}}{\tau}\right),
\end{aligned}
$$

for any $c>1 / 2$. Move the line of integration to $\operatorname{Re} s=0$; there is no pole of the integrand in the region $0<\operatorname{Re} s<c$, and it can be shown that the contribution from the horizontal lines is less than that from the new vertical line. The integral on $\operatorname{Re} s=0$ is estimated using (6.16) and 
$\left((8 N)^{i t}-N^{i t}\right) / t \ll \min (1,1 /|t|)$. Thus

$$
N\left(1 / 2+i t, \chi \lambda^{m}\right) \ll(W+\tau)^{1 / 3} \log ^{c} x+N^{1 / 2+\eta} / \tau,
$$

which is $\ll N^{1 / 2} x^{-4 \eta / 3}$ if we set $\tau=W$ and demand $x^{7 \eta / 3}<W<$ $N^{3 / 2} x^{-5 \eta}$. This latter upper bound is satisfied in our application where $N=x / 4 A B>x / 4 W^{2}$. So our final contribution to $R(A, B)$ is

$$
\begin{aligned}
& \ll l x^{1 / 2}\left(\frac{x}{A B}\right)^{1 / 2} x^{-4 \eta / 3} \\
& \times \sum_{\substack{\chi,|m|<W \\
(m, \chi) \neq\left(0, \chi_{0}\right)}} \int_{-T}^{T}\left|A\left(1 / 2+i t, \chi \lambda^{m}\right) B\left(1 / 2+i t, \chi \lambda^{m}\right)\right| d t \\
& \ll l x^{1-4 \eta / 3} \log ^{c} x,
\end{aligned}
$$

on using Lemma 6 . In all cases the bound is $\ll l x^{1-\eta}$ as required.

Using Lemma 9 we can set $D=\left(x^{1-3 \eta} W^{-5 / 3}\right)^{2}$ and $u=2 Q / D^{1 / 2}$. It is easily checked that $D>z^{2}$ and $u<(2 Q)^{1 / 3}$. We now examine $\Sigma_{\mathrm{II}}(Q)$ with these choices. We will require the following result.

LEMma 10.

$$
\begin{aligned}
& \sum_{|m|<W}\left|L\left(1 / 2+\gamma+i t, \chi \lambda^{m}\right)\right|^{2} \ll(W+|t|) \log ^{c}(W+|t|), \\
& \int_{-T}^{T}\left|L\left(1 / 2+\gamma+i t, \chi \lambda^{m}\right)\right|^{2} d t \ll(T+|m|) \log ^{c}(T+|m|),
\end{aligned}
$$

for some $c>0$ and where $t$ and $\gamma$ are real with $|\gamma| \ll 1 / \log (W+|t|)$ and $W+|t| \geq e$ say.

The inclusion of $\gamma$ above means that by the use of

$$
f^{\prime}(a)=\frac{1}{2 \pi i} \int f(z)(z-a)^{-2} d z,
$$

with the path of integration a circle centre $1 / 2+i t$, radius $1 / \log (W+|t|)$, we can deduce

$$
\sum_{|m|<W}\left|L^{\prime}\left(1 / 2+i t, \chi \lambda^{m}\right)\right|^{2} \ll(W+|t|) \log ^{c}(W+|t|),
$$

and similarly for the integral.

Sketch proof of Lemma 10. We apply the method of Ramachandra, [25]. The Mellin transform gives

$$
\left|L\left(1 / 2+\gamma+i t, \chi \lambda^{m}\right)\right|^{2} \ll\left|\sum_{\mathfrak{a}} e^{-N \mathfrak{a} / P} \chi \lambda^{m}(\mathfrak{a}) N \mathfrak{a}^{-1 / 2-\gamma-i t}\right|^{2}
$$




$$
\begin{aligned}
& +P^{-2(\gamma+2 / \log V)} g\left(-\gamma-\frac{2}{\log V}\right) \\
& \quad \times \int_{-\infty}^{\infty} \Gamma\left(-\gamma-\frac{2}{\log V}+i v\right)\left|\sum_{N \mathfrak{a}<P} \frac{\chi \lambda^{m}(\mathfrak{a})}{N \mathfrak{a}^{1 / 2+2 / \log V-i(v+t)}}\right|^{2} d v \\
& +P^{-2(1 / 2+\gamma+2 / \log V)} g\left(-\frac{1}{2}-\gamma-\frac{2}{\log V}\right) \\
& \quad \times \int_{-\infty}^{\infty} \Gamma\left(-\frac{1}{2}-\gamma-\frac{2}{\log V}+i v\right)\left|\sum_{N \mathfrak{a}>P} \frac{\chi \lambda^{m}(\mathfrak{a})}{N \mathfrak{a}^{1+2 / \log V-i(t+v)}}\right|^{2} d v \\
& +P^{1-2 \gamma} \log ^{2} P,
\end{aligned}
$$

where the last term occurs only if $m=0$ and $\chi=\chi_{0}$. Here $V=W+|t|$ and $P$ is to be chosen, though we can assume $P^{1 / \log V} \ll 1$, and

$$
g(u)=\int_{-\infty}^{\infty} \Gamma(u+i v) G^{2}\left(1 / 2+\gamma+u+i(t+v), \chi \lambda^{m}\right) d v,
$$

where $G\left(s, \chi \lambda^{m}\right)$ is the gamma factor in the functional equation $L\left(s, \chi \lambda^{m}\right)=$ $G\left(s, \chi \lambda^{m}\right) L\left(1-s, \bar{\chi} \lambda^{-m}\right)$. A version of (7.11) appears in Johnson [16], p. 199. Introducing the sum over $|m|<W$, we make use of Lemma 6 and

$$
G\left(s, \chi \lambda^{m}\right) \ll\left(m^{2}+(\operatorname{Im} s)^{2}\right)^{1 / 2-\sigma},
$$

which follows from Stirling's formula. Note that in the first term in (7.11), $e^{-N \mathfrak{a} / P}$ is arbitrarily small if $N \mathfrak{a}>P \log ^{2} P$ say, at which point the sum can be truncated. Then Lemma 6 will give, as it does for the second term in (7.11), a bound $\ll(W+P) \log ^{c} P$. Lemma 6 is not directly applicable to the infinite sum in the third term of (7.11). But using

$$
\left|\sum_{N \mathfrak{a}>P} \ldots\right|^{2} \leq\left(\sum_{\lambda=0}^{\infty} 2^{\lambda(\sigma-1)}\left|\sum_{2^{\lambda} P<N \mathfrak{a} \leq 2^{\lambda+1} P} \ldots\right|^{2}\right)\left(\sum_{\lambda=0}^{\infty} 2^{-\lambda(\sigma-1)}\right),
$$

valid for $\sigma>1$, Lemma 6 can be applied to each finite sum and the results combined to give a contribution $\ll P^{-2} V^{2}(W+P) \log ^{c} V$, the $V^{2}$ arising from the gamma factor. Thus

$\sum_{|m|<W}\left|L\left(1 / 2+\gamma+i t, \chi \lambda^{m}\right)\right|^{2} \ll\left(W+P+P^{-2} V^{2}(W+P)\right) \log ^{c} V \ll V \log ^{c} V$

(on choosing $P=V$ ), as required. For (7.10), use on (7.11) the classical mean value result

$$
\int_{-T}^{T}\left|\sum_{n} a_{n} n^{-i t}\right|^{2} d t \ll \sum_{n} a_{n}^{2}(n+T),
$$

in place of Lemma 5 . 
Returning to $\Sigma_{\mathrm{II}}(Q)$, we use (4.1) in writing

$$
R^{+}\left(\mathcal{A}_{\mathfrak{f h}}, D / 2 Q\right) \ll_{\varepsilon} \sum_{N \mathfrak{c}<D / 2 Q} C(\mathfrak{c}) r\left(\mathcal{A}_{\mathfrak{f h}}, \mathfrak{c}\right) \quad \text { for some }|C(\mathfrak{c})| \leq \sigma(\mathfrak{c}) .
$$

Then, just as we obtained (7.9), we have

$$
\begin{aligned}
& \Sigma_{\mathrm{II}}(Q) \\
& \quad \ll_{\varepsilon} l \sum_{\chi} \sum_{|m|<W}\left|\int_{1 / 2-i T}^{1 / 2+i T} \widehat{g}(s) E\left(s, \chi \lambda^{m}\right) C\left(s, \chi \lambda^{m}\right) L\left(s, \chi \lambda^{m}\right) d s\right|,
\end{aligned}
$$

where

$$
\begin{aligned}
= & \sum_{\substack{Q \leq N \mathfrak{f h}<2 Q \\
N \mathfrak{f}<u}}^{E\left(s, \chi \lambda^{m}\right)} A(\mathfrak{f}) B(\mathfrak{h}) \chi \lambda^{m}(\mathfrak{f h}) N(\mathfrak{f h})^{-s} \\
= & \frac{1}{2 \pi i} \int_{\substack{1 / 2+1 / \log x-i \tau_{2} \\
1 / 2+1 / \log x+i \tau_{1}}} A\left(s+w, \chi \lambda^{m}\right) B\left(s+w, \chi \lambda^{m}\right) \frac{(2 Q)^{w}-Q^{w}}{w} d w \\
& +O_{\eta}\left(\frac{Q^{1 / 2+\eta}}{\tau}\right),
\end{aligned}
$$

where $\tau=\min \left(\tau_{1}, \tau_{2}\right)$, having used Perron's Theorem. Since the coefficients $B(\mathfrak{h})$ are either all 1 or equal to $\log N \mathfrak{h}, B\left(s, \chi \lambda^{m}\right)$ is either $L\left(s, \chi \lambda^{m}\right)$ or $L^{\prime}\left(s, \chi \lambda^{m}\right)$.

We move the line of integration in (7.15) to $\operatorname{Re} w=1 / \log x$ and choose $\tau_{1}$ and $\tau_{2}$ of order $W$. From (7.10) we have for each $|m|<W$,

$$
\int_{W}^{2 W}\left|L\left(1 / 2+1 / \log x+i t, \chi \lambda^{m}\right)\right|^{2} d t \ll W \log ^{c} W,
$$

so there exists $\tau_{1} \in[W, 2 W]$ depending on $m$ such that

$$
L\left(1 / 2+1 / \log x+i \tau_{1}, \chi \lambda^{m}\right) \ll \log ^{c} W,
$$

for some $c>0$, the same conclusion holding for $L^{\prime}\left(s, \chi \lambda^{m}\right)$. By convexity this bound holds on the half-line to the right of $1 / 2+1 / \log x$. Similarly, there is a $\tau_{2} \in[-2 W,-W]$ with this same bound. Thus the contribution to $(7.15)$ of the horizontal lines is $\ll\left(u^{1 / 2}+Q^{1 / 2}\right) W^{-1} \log ^{c} x$ which, since $u<Q^{1 / 2}$, is dominated by the error in (7.15). This in turn is $\ll 1$ because $W>x^{1 / 3}>x^{1 / 4+\eta / 2}>Q^{1 / 2+\eta}$. Hence the error from (7.15) contributes in $(7.14)$ 


$$
\begin{aligned}
& \ll l x^{1 / 2} \sum_{\chi} \int_{-T}^{T}\left(\sum_{|m|<W}\left|C\left(1 / 2+i t, \chi \lambda^{m}\right)\right|^{2}\right)^{1 / 2} \\
& \times\left(\sum_{|m|<W}\left|L\left(1 / 2+i t, \chi \lambda^{m}\right)\right|^{2}\right)^{1 / 2} d t \\
& \ll l x^{1 / 2} T\left(W+D Q^{-1}\right)^{1 / 2}(W+T)^{1 / 2} \log ^{c} x \\
& \ll l x^{1 / 2}\left(W+W^{1 / 2} D^{1 / 2} Z^{-1 / 2}\right) \log ^{c} x \ll l x^{1-\eta}
\end{aligned}
$$

having substituted for $D, Z$ and used $x^{1 / 3}<W<x^{3 / 8-5 \eta / 4}$.

Substituting the main term, obtained from (7.15) on moving the line of integration, into (7.14) gives

$$
\begin{aligned}
& \ll l x^{1 / 2} \sum_{\chi} \int_{1 / \log x-2 i W}^{1 / \log x+2 i W} \frac{(2 Q)^{w}-Q^{w}}{w} d w \\
& \times \int_{1 / 2-i T}^{1 / 2+i T} \sum_{|m|<W}\left|A\left(s+w, \chi \lambda^{m}\right) B\left(s+w, \chi \lambda^{m}\right) C\left(s, \chi \lambda^{m}\right) L\left(s, \chi \lambda^{m}\right) d s\right| .
\end{aligned}
$$

Using the order result (6.16) and Cauchy-Schwarz, the sum in the inner integral is bound by

$$
\begin{gathered}
W^{1 / 3}\left(\sum_{|m|<W}\left|A\left(s+w, \chi \lambda^{m}\right) C\left(s, \chi \lambda^{m}\right)\right|^{2}\right)^{1 / 2}\left(\sum_{|m|<W}\left|B\left(s+w, \chi \lambda^{m}\right)\right|^{2}\right)^{1 / 2} \\
\ll W^{1 / 3}(W+u(D / Q))^{1 / 2}(W+|\operatorname{Im}(s+w)|)^{1 / 2} \log ^{c} x .
\end{gathered}
$$

Yet $\operatorname{Im}(s+w) \ll W+\log ^{4} x$ while $u(D / Q) \leq 2 D^{1 / 2}$, so the bound is

$$
\ll\left(W^{4 / 3}+W^{5 / 6}\left(x^{1-3 \eta} W^{-5 / 3}\right)^{1 / 2}\right) \log ^{c} x \ll x^{1 / 2-5 \eta / 4} \log ^{c} x,
$$

which in (7.16) gives a bound $\ll l x^{1-\eta}$. Thus $\Sigma_{\mathrm{II}}(Q) \ll l x^{1-\eta}$.

Combining, within (6.2), these results on the sieve with Proposition 8 gives

$$
\sum_{\mathfrak{p}} \theta(\mathfrak{p}) \geq \frac{2 l x}{h(\mathfrak{f}) \log x}\left\{H(z, Z, D)-c \varepsilon+O\left(\frac{1}{\log x}\right)\right\}+O_{\varepsilon_{1} \varepsilon, \eta}\left(l x^{1+\varepsilon_{1}-\eta}\right),
$$

where

$$
\begin{aligned}
H(z, Z, D)= & e^{-\gamma} \frac{\log x}{\log z} f\left(\frac{\log D}{\log z}\right)-\int_{\log z / \log x}^{\log Z / \log x} \frac{d t}{t(1-t)} \\
& -3 e^{-\gamma} F(3) \int_{\log Z / \log x}^{1 / 2} \frac{d t}{t\left(\frac{\log D}{\log x}-t\right)},
\end{aligned}
$$


having used partial summation in the sum over prime ideals in (7.5). The above result is, by Proposition 8 and Lemma 9, valid for $x^{-3 / 8+\eta}<l<$ $x^{-1 / 3}$. Substituting for $z, Z$ and $D$, writing $l=x^{-\theta}$ and using

$$
f(u)=\frac{2 e^{\gamma}}{u} \log (u-1), \quad 2 \leq u \leq 4,
$$

and

$$
F(u)=\frac{2 e^{\gamma}}{u}, \quad 0<u \leq 3,
$$

gives $H(z, Z, D) \geq c(\theta)-C_{1} \varepsilon-C_{2} \eta$ where

$$
c(\theta)=\frac{3}{3-5 \theta} \log \left(\frac{(6-13 \theta)(9-20 \theta)}{9 \theta}\right)-\log \left(\frac{(3-5 \theta)(1-\theta)}{5 \theta^{2}}\right) .
$$

It is easily checked that $c(\theta)>0.0021$ for $1 / 3 \leq \theta \leq 0.33691<3 / 8$, so on choosing $\eta, \varepsilon$ and $\varepsilon_{1}$ sufficiently small

$$
\sum_{\mathfrak{p}} \theta(\mathfrak{p}) \geq \frac{1}{500} \frac{2 l x}{h(\mathfrak{f}) \log x}
$$

for $x^{-1 / 3}>l \geq x^{-0.3369}$ as required.

8. Concluding remarks. The range of validity of Theorem 2 can be extended to $l>x^{-1 / 3} \log ^{3} x$ by choosing $z=x^{1 / 3}, Z=x^{1-\varepsilon} W^{-5 / 3}$. It is easily checked that $H(z, Z, D)>0$.

Lemma 6 should be compared with Theorem 6.2 of [3] that states that

$$
\sum_{|m|<W} \int_{-T}^{T}\left|\sum_{N \mathfrak{a}<X} \mathfrak{c}(\mathfrak{a}) \lambda^{m} \chi(\mathfrak{a}) N \mathfrak{a}^{-i t}\right|^{2} d t \ll\left(X+W^{2}+T^{2}\right) \sum_{N \mathfrak{a}<X}|\mathfrak{c}(\mathfrak{a})|^{2} .
$$

From the geometry of the problem considered in [3], $W$ and $T$ are of the same magnitude and thus $W^{2}+T^{2}$ is a fair reflection of the size of the region $|m|<W,|t|<T$. In our present problem, where $T$ is so much smaller than $W$, this is not the case and so it is advantageous to use Lemma 6 . Unfortunately, $W^{2}+T^{2}$ still occurs, primarily from the order result (6.16) and the gamma factor (7.13).

Comparing the methods of this paper with those of [15] we see we have made no use of the Halász-Montgomery inequality. Such a result has been given for a quadratic imaginary number field as Lemma 7.2 of [3]. It is seen from there that the inequality depends on the order of Hecke $L$-functions on various vertical lines, and so will include the factor $W^{2}+T^{2}$. Because of this, the Halász-Montgomery inequality affords a far smaller advantage over Lemma 6 than it does in the rational case or even in [3]. But further, without this inequality we cannot follow [8] in looking at a decomposition more complicated than (6.2) nor use the observation that Lemma 3 gives bounds for $W^{ \pm}(\mathcal{A}, u, z)$, not just $S(\mathcal{A}, z)$. 


\section{References}

[1] N. C. Ankeny, Representations of primes by quadratic forms, Amer. J. Math. 74 (1952), 913-919.

[2] K. Bulota, On Hecke Z-functions and the distribution of the prime numbers of an imaginary quadratic field, Litovsk. Mat. Sb. 4 (1964), 309-328 (in Russian).

[3] M. D. Coleman, The distribution of points at which binary quadratic forms are prime, Proc. London Math. Soc. (3) 61 (1990), 433-456.

[4] -, A zero-free region for the Hecke L-functions, Mathematika 37 (1990), 287-304.

[5] - , The distribution of points at which norm-forms are prime, J. Number Theory 41 (1992), 359-378.

[6] P. X. Gallagher, A large sieve density estimate near $\sigma=1$, Invent. Math. 11 (1970), 329-339.

[7] H. Halberstam and H. E. Richert, Sieve Methods, Academic Press, London, 1974.

[8] D. R. Heath-Brown and H. Iwaniec, On the differences between consecutive primes, Invent. Math. 55 (1979), 49-69.

[9] D. R. Heath-Brown and S. J. Patterson, The distribution of Kummer sums at prime arguments, J. Reine Angew. Math. 310 (1979), 111-130.

[10] E. Hecke, Eine neue Art von Zeta Functionen und ihre Beziehungen zur Verteilung der Primzahlen, I, II, Math. Z. 1 (1918), 357-376; 6 (1920), 11-51.

[11] J. G. Hinz, A generalization of Bombieri's prime number theorem to algebraic number fields, Acta Arith. 51 (1988), 173-193.

[12] —, Chen's theorem in totally real algebraic number fields, ibid. 58 (1991), 335-361.

[13] H. Iwaniec, Rosser's sieve, ibid. 36 (1980), 171-202.

[14] -, A new form of the error term in the linear sieve, ibid. 37 (1980), 307-320.

[15] H. Iwaniec and M. Jutila, Primes in short intervals, Ark. Mat. 17 (1979), 167176 .

[16] D. Johnson, Mean values of Hecke L-functions, J. Reine Angew. Math. 305 (1979), 195-205.

[17] W. B. Jurkat and H.-E. Richert, An improvement of Selberg's sieve method, I, Acta Arith. 11 (1965), 217-240.

[18] R. M. Kaufman, Estimate of the Hecke L-function on the half-line, Zap. Nauchn. Sem. Leningrad. Otdel. Mat. Inst. Steklov. (LOMI) 91 (1979), 40-51 (in Russian).

[19] F. B. Koval'chik, Density theorems and the distribution of primes in sectors and progressions, Dokl. Akad. Nauk SSSR (N.S.) 219 (1974), 31-34 (in Russian).

[20] J. P. Kubilius, The decomposition of prime numbers into two squares, ibid. 77 (1951), 791-794 (in Russian).

[21] - On some problems of the geometry of prime numbers, Mat. Sb. (N.S.) 31 (1952), 507-542 (in Russian).

[22] - On a problem in the $n$-dimensional analytic theory of numbers, Viliniaus Valst. Univ. Mokslo dardai Fiz. Chem. Moksly Ser. 4 (1955), 5-43.

[23] T. Mitsui, Generalised prime number theorem, Japan. J. Math. 26 (1956), 1-42.

[24] R. W. K. Odoni, The distribution of integral and prime-integral values of systems of full-norm polynomials and affine-decomposable polynomials, Mathematika 26 (1979), 80-87.

[25] K. Ramachandra, A simple proof of the mean fourth power estimate for $\zeta(1 / 2+i t)$ and $L(1 / 2+i t, \chi)$, Ann. Scuola Norm. Sup. Pisa Cl. Sci. 1 (1974), 81-97.

[26] S. Ricci, Local distribution of primes, Ph.D. thesis, University of Michigan, 1976.

[27] H.-E. Richert, Selberg's sieve with weights, Mathematika 16 (1969), 1-22. 
[28] G. J. Rieger, Verallgemeinerung der Siebmethode von A. Selberg auf algebraische Zahlkörper III, J. Reine Angew. Math. 208 (1961), 79-90.

[29] W. Schaal, Obere und untere Abschätzungen in algebraischen Zahlkörpern mit Hilfe des linearen Selbergschen Siebes, Acta Arith. 13 (1968), 267-313.

[30] E. C. Titchmarsh, The Theory of the Riemann Zeta-function, Oxford University Press, 1951.

[31] R. C. Vaughan, An elementary method in prime number theory, Acta Arith. 37 (1980), 111-115.

DEPARTMENT OF MATHEMATICS

UMIST

P.O. BOX 88

MANCHESTER, M60 1QD

ENGLAND 